\title{
Forme de la tige, tarifs de cubage et ventilation de la production en volume chez le Chêne sessile
}

\author{
Jean-François Dhôte*, Elvire Hatsch et Daniel Rittié \\ Équipe « Dynamique des Systèmes Forestiers », Unité Associée ENGREF/INRA de Sciences Forestières, \\ 14 rue Girardet, 54042 Nancy Cedex, France
}

(Reçu le 5 juin 1998 ; accepté le $1^{\text {er }}$ septembre 1999)

\begin{abstract}
Résumé - Bien que des tarifs de cubage à double entrée existent depuis 20 ans pour les principales essences feuillues françaises, les volumes ainsi estimés pour le Chêne sessile apparaissent parfois trop forts aux gestionnaires forestiers. Nous abordons cette question en analysant la production en volume des chênaies. L'analyse est conduite en modélisant d'une part la répartition du volume entre tige et branches, d'autre part la forme géométrique de la tige. La robustesse de la méthode est testée grâce à plusieurs jeux de données de grande taille, couvrant les principales sources de variabilité (âge, sylviculture). La cohérence avec les tarifs de cubage existants est évaluée favorablement. Des simulations basées sur un modèle de croissance permettent d'explorer les variations de la production selon la fertilité de la station et la sylviculture, mais aussi de décomposer cette production selon divers critères (tige-branches, houppiergrume, écorce-aubier-duramen) et finalement d'évaluer le rendement du Chêne lors de la transformation industrielle. Les résultats indiquent que le désaccord entre praticiens et chercheurs sur les volumes porte tout simplement sur l'objet cubé (volume commercial vs. total).
\end{abstract}

tarif de cubage / profil de tige / production en volume / stratégie sylvicole / Quercus petraea (Matt.) Liebl.

\begin{abstract}
Stem taper curves, volume tables and volume yield compartments in Sessile Oak. Since the 1970ies, efficient volume tables are available for the main broadleaved species. Nevertheless, estimates of volumes obtained this way for Sessile Oak are still considered too high by some forest managers. We address this question by analysing the volume yield of Oak stands. The analysis is done by modelling the distribution of volume between stem and branches and the geometrical shape of stems (taper curves). The robustness of the method was tested by use of several, large size samples, covering the main sources of variability (age and silviculture). The coherence with former volume tables was favourably tested. Simulations based on a growth model were done in order 1) to explore the variability of volume yield with respect to site quality and silvicultural scenarios; 2) to dispatch total yield according to different criteria (stem-branches, crown-log, bark-sapwood-heartwood); 3) to evaluate the percentage of losses during industrial processing of Oak timber. Results indicate that the disagreement between practicians and scientists on volume simply refers to the nature of the volume (commercial log vs. total volume).
\end{abstract}

volume table / taper curve / volume yield / silvicultural strategies / Quercus petraea (Matt.) Liebl.

\section{INTRODUCTION}

Au cours des 10 dernières années, un important effort de recherche sur les Chênes sessile et pédonculé a été consenti par la plupart des organismes forestiers français (Bull. Techn. ONF No. 33, 1997). Concernant la sylviculture et la dynamique des peuplements de Chêne sessile (production, croissance individuelle,

* Correspondance et tirés à part

Tel. 03833968 46; Fax. 03833273 81; e-mail: dhote@ nancy-engref.inra.fr 
morphologie des arbres), ces recherches ont abouti à la construction d'un modèle de croissance $[7,17]$. Une partie de ces connaissances, maintenant disponibles dans la plate-forme logicielle Capsis [9], ont déjà fait l'objet d'applications opérationnelles [1].

Parmi ces recherches, celle consacrée à la forme de la tige nous a semblé mériter une diffusion particulière. En effet, la forme plus ou moins conique ou cylindrique de la grume a de fortes répercussions sur l'apparence esthétique des débits (dessin des arcatures sur plots ou feuilles de placages) et sur le rendement-matière lors de la première transformation (taux de déchets). En deuxième lieu, la forme finale de la tige doit être vue comme le résultat de l'accumulation progressive des cernes annuels ; par conséquent, le thème de la forme est lié étroitement à celui de la largeur des cernes, dont on connaît l'influence sur la plupart des propriétés du matériau-bois, notamment chez le Chêne [16, 22].

Enfin, la forme de la tige est liée à la question de la validité des tarifs de cubage, à leur adéquation dans diverses conditions de sylviculture. L'estimation du volume des arbres forestiers est une question qui reste assez sensible, malgré les travaux de recherche et le développement de tarifs de cubage réalisés depuis les années 1970, tant à l'ONF qu'à l'INRA (voir, pour le Chêne, [4]). Ce constat vaut tout particulièrement pour les essences feuillues : les découpes à retenir, tout simplement pour définir l'objet cubé, varient avec la grosseur de l'arbre ; le volume total d'un arbre mûr se décompose en parties de valeurs marchandes très inégales (bille de pied, surbilles, houppier). On a souvent l'impression que le critère de l'exactitude numérique passe au second plan, dans la gestion courante, derrière le besoin d'annoncer des volumes correspondant au mieux aux demandes des acheteurs immédiats. Les considérations d'ordre commercial sont donc omniprésentes, comme le dit si bien l'expression «tarifs de cubage».

Il est a priori du ressort du gestionnaire forestier, et non d'un Institut de Recherche, de choisir la méthode de cubage appropriée, lors des ventes ou des inventaires d'aménagement. Toutefois, des comparaisons entre les cubages «gestion» et «recherche» sont inévitables, soit lors de l'utilisation de simulateurs de croissance, soit lorsque des données de terrain comme les prix du mètre cube sont utilisées en recherche appliquée. De façon insistante, au cours des dernières années, nos prévisions de production en volume pour les chênaies ont été jugées beaucoup trop optimistes par des collègues de l'Office National des Forêts (discussions informelles suite au stage [1]). Pour éviter que ce débat n'ôte leur crédibilité aux modèles aux yeux de ceux qui sont censés en utiliser les résultats, il nous a semblé important de revenir sur la question du cubage du Chêne, à partir d'une modélisation de la forme des tiges.

Il fallait aussi s'assurer de la validité du tarif de J. Bouchon pour des chênes de morphologie «trapue», qu'on trouve actuellement en taillis-sous-futaie mais qui pourraient devenir plus abondants même en futaie, si les forestiers s'orientent vers des régimes d'éclaircies plus fortes [14]. Enfin, en matière de cubage, les méthodes de simulation dont nous disposons nous permettent de faire beaucoup plus qu'une simple estimation du volume boisfort : si l'on sait prédire la forme externe de la tige et sa géométrie interne (l'écorce, les cernes, l'aubier, le noyau central non élagué), on peut calculer très simplement un grand nombre de volumes.

Les objectifs poursuivis dans cet article sont donc, d'une part, d'apporter des arguments objectifs contre les reproches de surestimation faits par certains gestionnaires forestiers aux tarifs de cubage «recherche», d'autre part d'illustrer l'intérêt des méthodes de modélisation pour l'orientation stratégique de la sylviculture des chênaies. Pour cela, nous avons besoin d'un simulateur de la croissance individuelle, sous diverses hypothèses de fertilité de la station et de sylviculture : nous rappelerons, dans les grandes lignes, le contenu de ce simulateur.

Nous présenterons plus en détail une méthode de simulation de la forme des tiges de Chêne sessile. Nous décrirons comment a été établie et validée une équation décrivant la variation du diamètre le long de la tige, ainsi que les relations annexes qui permettent de déformer et d'adapter cette équation à la plupart des morphologies rencontrées (arbres trapus en TSF, plus ou moins élancés en futaie). Nous montrerons la cohérence entre les calculs de volume tirés de cette équation et ceux fournis par le tarif à deux entrées de [4].

Enfin, nous aborderons les utilisations de ces résultats à l'échelle du peuplement, en étudiant la ventilation de la production d'une chênaie par catégories de produits (petits bois, bois moyens, gros bois), selon différents modes de découpe (bois-fort, grume commerciale) et selon différents compartiments.

\section{MATÉRIEL ET MÉTHODES}

L'analyse de la forme a été menée grâce à deux échantillons de conceptions différentes. Le premier a été acquis dans le cadre du Contrat INRA-ONF «Sylviculture et qualité du bois de Chêne sessile»199296 ; il comprend un petit nombre d'arbres étudiés très finement par analyse de tige (étant donnée la longévité du Chêne, et par conséquent le nombre énorme de cernes à mesurer, une telle étude ne peut être envisagée que 
pour quelques dizaines d'arbres). Le second échantillon est constitué de plusieurs milliers de fiches de cubage recueillies en futaie régulière, dans le réseau des placettes permanentes suivies par l'INRA. Dans ce cas, seul la forme précise de la tige, à une date donnée, est disponible.

\subsection{Les 82 Chênes sessiles adultes de la Convention INRA-ONF}

Cette collection résulte d'un échantillonnage stratifié dans l'aire de distribution de l'essence. Elle est répartie en 5 régions : les collines sous-vosgiennes et la plaine alsacienne, le Plateau lorrain, l'Allier-Bourbonnais, la moyenne Vallée de la Loire, les collines de BasseNormandie. Dans chaque région est représentée une large gamme de stations, regroupées en 3 classes (bonne, moyenne et pauvre). Dans chaque combinaison régionstation, nous avons retenu des peuplements appartenant à la futaie régulière serrée et au taillis-sous-futaie. Cet échantillonnage visait à bien décrire les sources de variabilité de l'essence et à fournir des exemples d'arbres possédant des vitesses de croissance radiale et des morphologies aussi variées que possible. Une caractérisation plus complète de cet échantillon peut être trouvée dans [8].

Les 82 chênes retenus sont âgés de 61 à 224 ans (moyenne 153), ont des diamètres compris entre 42 et $104 \mathrm{~cm}$ (moyenne 62) et des hauteurs comprises entre 17 et $40 \mathrm{~m}$ (moyenne 28). La largeur des cernes moyenne à $1,30 \mathrm{~m}$ est comprise entre 1,26 et $3,95 \mathrm{~mm}$, avec une prédominance des arbres à cernes fins (moyenne $1,95 \mathrm{~mm}$ ). 47 arbres sont issus de futaie et 35 de taillis sous futaie.

\subsection{Les 2948 fiches de cubage Chêne}

Nous avons conçu et saisi un sous-échantillon des fiches de cubage recueillies dans les placettes permanentes de Chêne. Les principes de ce rééchantillonnage étaient d'une part de couvrir une large gamme d'âges (40 à 220 ans), d'autre part de privilégier les essais d'éclaircie comprenant des modalités bien différenciées en termes de densité, afin de détecter un éventuel effet sylvicole sur la forme des tiges. À cet effet, nous avons retenu plusieurs placettes intéressantes à divers titres, soit très vieilles, soit très claires ou très denses (Ducellier, Sablonnières Rouges et Launay-Morel à Bellême, Allées de Blois et Charmaie à Blois, Clé des Fossés, Richebourg et Morat à Tronçais), dans des peuplements de 75 à 210 ans. Nous avons aussi considéré les 3 essais d'éclaircie de Blois (Sablonnières, Pauverts,
Marchais des Cordeliers), les 2 de Champenoux (Butte de Tir et Grande Bouzule) et le Trésor à Tronçais. Les effectifs sont donc déséquilibrés : 334 arbres à Bellême contre 1492 (Blois), 468 (Champenoux) et 654 (Tronçais). Dans chacun des 98 lots de fiches de cubage (une placette à une date), nous avons conservé au maximum 40 fiches (en moyenne 30), distribuées uniformément dans le nuage de points (hauteur, diamètre). La circonférence (resp. la hauteur) moyenne de ces 98 lots s'étale de 30 à $250 \mathrm{~cm}$ (resp. 10 à $35 \mathrm{~m}$ ).

\subsection{Un modèle pour le profil de la tige}

Dans des travaux précédents [12], nous avions montré que le profil d'une tige de Chêne sessile, c'est-à-dire la variation longitudinale du diamètre sous ou sur écorce, peut être correctement assimilé à une succession de deux troncs de cône (figure 1). Le cône supérieur correspond au houppier ; le tronc de cône inférieur, décrivant la tige nette de branches, doit être corrigé vers le sol par une fonction convexe pour rendre compte de l'empattement basal. La jonction entre les deux troncs de cône peut être continue ou discontinue. Dans le deuxième cas, il existe une variation brutale du diamètre de la section, qui coïncide en général avec l'insertion d'une fourche ou bien d'un faisceau de plusieurs branches vigoureuses. Pour décrire mathématiquement cette morphologie, nous

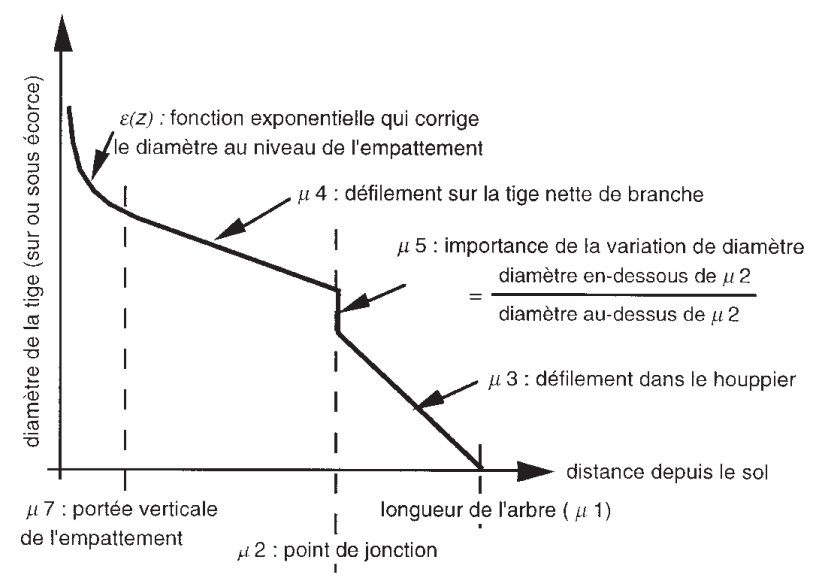

Figure 1. Modèle du profil longitudinal de la tige de Chêne sessile. La signification des 7 paramètres $\mu_{i}$ est indiquée. 
avons construit le modèle suivant, dont les 7 paramètres possèdent tous un sens forestier concret :

$d(z)=\mu_{3} *\left(\mu_{1}-z\right)$ si $\mu_{2}<z<\mu_{1}$ (dans le houppier)

$d(z)=\left(\mu_{4} *\left(\mu_{2}-z\right)+\mu_{5} * \mu_{3} *\left(\mu_{1}-\mu_{2}\right)\right) *(1+\varepsilon(z))$ si 0 $\leq z \leq \mu_{2}$ (tige nette de branches)

$\operatorname{avec} \varepsilon(z)=\mu_{6}^{*} e^{-z / \mu_{7} * \operatorname{Ln}\left(100 * \mu_{6}\right)}$

où

$d(z)$ diamètre de la tige (sur ou sous écorce) au niveau $z$ au-dessus du sol $(\mathrm{cm})$

$\mu_{1} \quad$ sommet du profil $(\mathrm{m})$, légèrement inférieur à la hauteur totale de l'arbre,

$\mu_{2}$ estimation du point de jonction tige-houppier (m),

$\mu_{3}$ décroissance métrique $(\mathrm{cm} / \mathrm{m})$ dans le houppier,

$\mu_{4}$ décroissance métrique $(\mathrm{cm} / \mathrm{m})$ le long de la tige,

$\mu_{5}$ amplitude du saut de diamètre à la base du houppier ( $\geq 1,1$ si pas de saut),

$\varepsilon(z)$ fonction exponentielle corrigeant le tronc de cône dans l'empattement,

$\mu_{6}$ «empattement en diamètre », c'est-à-dire valeur de la correction en $z=0$,

$\mu_{7} \quad$ portée verticale de l'empattement, en mètres (c'est-à-dire le niveau où $\varepsilon(z)$ vaut $1 \%$ ).

Pour ajuster ce modèle, nous utilisons la méthode des moindres carrés non-linéaires. L'algorithme de minimisation est celui de Gauss-Marquardt. Le programme fournit l'ensemble des statistiques utiles : écart-type résiduel, coefficient de détermination $\left(R^{2}\right)$, valeur estimée des paramètres, estimation de leur erreur et de la matrice des corrélations entre paramètres. Il renvoie une image des données et de la courbe ajustée. L'appréciation visuelle de la qualité de l'ajustement est très importante.

Le modèle [1] a été, dans un premier temps, mis au point et ajusté sur les données de l'échantillon INRAONF (cubage exterme actuel et profils internes mesurés par analyse de tige : [12]). Nous présentons dans cet article l'étape suivante de la recherche, qui consiste à modéliser les variations des 7 paramètres de l'équation (1) en fonction de descripteurs simples de l'arbre (âge, hauteur, diamètre, base du houppier). Pour cela, nous utiliserons le jeu de données fourni par les 2948 fiches de cubage, qui sera scindé en deux parties, l'une pour l'ajustement, l'autre pour la validation.

\subsection{Estimation des compartiments dans l'arbre (écorce, aubier-duramen, cœur branchu)}

Pour convertir un diamètre sur-écorce en son équivalent sous-écorce, nous appliquons une fraction fixe de 0,926. Cette valeur a été obtenue en comparant les cir- conférences à $1,30 \mathrm{~m}$ sur et sous écorce mesurées sur les analyses de tige de l'échantillon INRA-ONF. Il s'ensuit que la largeur des cernes est inférieure de 7,4\% à l'accroissement radial mesuré sur écorce.

Pour estimer l'importance de l'aubier, nous appliquons les résultats tirés de l'échantillon INRA-ONF. Nous avions montré [12] que le nombre de cernes d'aubier est uniforme le long de la tige ; il s'établit à une valeur caractéristique pour chaque arbre, valeur prédite de la manière suivante :

$$
\begin{gathered}
r h=0,3161 \cdot d_{130}{ }^{0,976} \cdot h^{-0,344} \\
n b c \_ \text {Aubier }=6,1+0,382 \cdot \frac{r h}{l c \_ \text {moy_Aubier }}
\end{gathered}
$$

où $r h$ est le rayon du houppier $(\mathrm{m})$ prédit en fonction du diamètre $d_{130}(\mathrm{~cm})$ et de la hauteur totale $h(\mathrm{~m})$ $n b c \_$Aubier le nombre de cernes d'aubier et lc_moy_Aubier la largeur moyenne des cernes dans l'aubier.

On remarquera que l'aubier est présent dans les deux termes de l'équation (3), qui est donc implicite. Pour la résoudre numériquement, nous partons du cerne le plus récent et ajoutons les cernes un par un, jusqu'à ce que le critère (3) soit réalisé. L'équation (2) a été ajustée à partir de plus d'un millier de mesures réalisées par [3] dans des futaies régulières à divers stades ; son bon comportement pour des arbres de taillis-sous-futaie a été testé [15]. L'équation (3) a été ajustée pour les 82 arbres de l'échantillon INRA-ONF [17].

L'importance du cœur branchu (noyau central nonélagué de la tige) est estimée grâce à la position, à chaque pas de temps, de la base du houppier : sa trace à l'intérieur de la grume est considérée comme la limite de la présence de nœuds. Cette procédure revient à supposer que les branches, dès qu'elle meurent, s'élaguent physiquement. Cette hypothèse est suffisante pour des branches fines, constituées uniquement d'aubier, mais trouve ses limites pour de grosses branches duraminisées. Il faudrait donc, pour mieux faire, pouvoir estimer le diamètre et le degré de duraminisation des branches, ce que nous nous proposons de réaliser ultérieurement.

Pour estimer la position de la base du houppier, nous utilisons une équation allométrique qui sera présentée plus loin dans cet article (équation (10)).

\subsection{Intégration dans un modèle de croissance}

Pour simuler, nous avons besoin non seulement des méthodes d'estimation des volumes exposées plus bas, 
mais aussi d'un modèle de croissance de type «modèle d'arbre indépendant des distances ». L'ensemble des méthodes sont rassemblées dans le logiciel Fagacées. Ce simulateur gère un peuplement d'un hectare décrit par une liste d'arbres et permet de manipuler celle-ci graphiquement selon diverses méthodes d'éclaircie ; il calcule toutes les statistiques de peuplement usuelles ; en outre, il sauvegarde la trajectoire (diamètre, hauteur, base du houppier etc.) de la population des $N$ plus gros arbres restant en fin de révolution. $N$ peut être ajusté librement par l'utilisateur ; en pratique, nous avons choisi $N=70$, soit le nombre moyen d'arbres envisagés comme population-objectif.

Le modèle utilisé est composé de plusieurs modules. En premier lieu, la croissance en hauteur dominante est prédite d'après le modèle de [10], version France entière. Nous avons supposé que la croissance en hauteur du Chêne sessile était indépendante de la sylviculture tant que l'indice de densité Rdi restait supérieur à 0,5 . Pour $R d i$ inférieur à 0,5 , la croissance donnée par les courbes de [10] est alors considérée comme un potentiel de la station ; la croissance réelle en hauteur s'en déduit en diminuant le potentiel proportionnellement à la densité. L'équation considérée est donc la suivante :

si $R d i>0,5$, alors $\frac{\Delta H_{0}}{\Delta t}=f_{\mathrm{H}}(\hat{a} g e$, indice_fertilité $)$

cf. équation (10). (4)

sinon $\frac{\Delta H_{0}}{\Delta t}=f_{\mathrm{H}}(\hat{a} g e$, indice_fertilité $) \cdot 2 \cdot R d i$

où $\frac{\Delta H_{0}}{\Delta t}$ est l'accroissement annuel en hauteur dominante (m/an) entre les âges $t$ et $t+\Delta t$.

La seconde étape consiste à prédire l'accroissement du peuplement en surface terrière. Celui-ci dépend de la hauteur dominante à l'âge considéré, de son accroissement et de l'indice de densité Rdi. L'équation a été construite et ajustée dans le cadre du Contrat INRA-ONF «Sylviculture et qualité du bois de Chêne» [17]. Dans le cas où la hauteur est affectée par la sylviculture, nous utilisons ici la hauteur dominante potentielle :

$$
\frac{\Delta G}{\Delta t}=\left(\mu_{1}+\mu_{2} \cdot \frac{\Delta H_{0}}{\Delta t}-\mu_{3} \cdot H_{0}\right) \cdot \frac{\left(\mu_{4}+1\right) \cdot R d i}{\mu_{4}+R d i}
$$

où $\frac{\Delta G}{\Delta t}$ et $\frac{\Delta H_{0}}{\Delta t}$ sont les accroissements annuels en surface terrière $\left(\mathrm{m}^{2} / \mathrm{ha} / \mathrm{an}\right)$ et en hauteur dominante (m/an) entre les âges $t$ et $t+\Delta t, H_{0}$ est la hauteur dominante moyenne entre ces deux âges (m), Rdi l'indice de densité calculé pour toutes les tiges (quel que soit leur étage de végétation), avec des paramètres prenant les valeurs suivantes : $\mu_{1}=0,27190 ; \mu_{2}=2,8018 ; \mu_{3}=$ $0,59684 \cdot 10^{-2} ; \mu_{4}=0,21852$.

Nous répartissons ensuite l'accroissement peuplement prédit par (5) entre tous les arbres, selon le modèle structurel suivant :

$$
\frac{\Delta g}{\Delta t}=\operatorname{Max}(0, \gamma \cdot(c-\sigma))
$$

où $c$ est la circonférence $(\mathrm{cm})$ de l'arbre à l'âge $t, \frac{\Delta g}{\Delta t}$ son accroissement en surface terrière $\left(\mathrm{cm}^{2} / \mathrm{an}\right)$ entre les âges $t$ et $t+\Delta t, \gamma$ et $\sigma$ deux paramètres variables dans le temps.

Pour calculer les paramètres $\gamma$ et $\sigma$, appelés respectivement «efficacité maximale» et «seuil», nous utilisons d'abord la relation (7) qui permet de fixer $\gamma$ :

$$
\gamma=0,0639+0,010 \cdot H_{0}(100) \text { (unité cm/an) }
$$

où l'indice de fertilité $H_{0}(100)$ est la hauteur dominante à 100 ans (m).

Le paramètre seuil $\sigma$ est finalement calculé de telle sorte que la somme des accroissements en surface terrière de tous les arbres soit égale au modèle de peuplement (5). Cette étape du calcul est purement numérique (il n'y a pas d'équation explicite donnant $\sigma$ ).

Pour en terminer avec la prévision des variables élémentaires, nous avons encore besoin d'estimer la hauteur individuelle des arbres. Ce qui est fait, connaissant leur diamètre, au moyen de courbes hauteur-diamètre à chaque pas de temps. Ces courbes sont astreintes à passer par les points $[0 \mathrm{~cm} ; 1,30 \mathrm{~m}]$ et $\left[D_{0} ; H_{0}\right]$ (couple diamètre dominant, hauteur dominante). Les courbes retenues sont des hyperboles dont deux paramètres sont fixes et le troisième (asymptote horizontale supérieure, qu'on peut considérer comme la hauteur maximale) est ajusté pour passer par le point $\left[D_{0} ; H_{0}\right]$. La méthode a été exposée dans [7].

Avec l'âge, le diamètre et la hauteur de chaque arbre, nous avons tous les éléments pour estimer leur profil de tige, d'après le modèle (1) et les équations (9) à (13), qui seront présentées plus bas. Cette méthode est utilisée pour cuber le volume géométrique de chaque tige à des découpes quelconques. En pratique, nous avons considéré les découpes suivantes :

- le volume de la tige à la découpe bois-fort $(7 \mathrm{~cm})$;

- le volume de la tige à la découpe $20 \mathrm{~cm}$;

- le volume-grume de la tige, défini ici comme le volume géométrique de la portion de tige dont le 
diamètre est supérieur à $20 \mathrm{~cm}$ et située sous le premier défaut (assimilé à la base du houppier).

En outre, nous avons calculé le volume de bois-fort total (tige et branches) par une nouvelle équation, développée spécifiquement pour ce travail (voir, plus bas, l'équation [15]).

\subsection{L'indice de densité $R d i$}

Pour définir des itinéraires sylvicoles, nous utiliserons un indice de densité des peuplements construit sur le principe de la loi d'autoéclaircie [24]. On a coutume de rendre compte de la mortalité naturelle par effet de compétition, dans des peuplements purs réguliers et très denses, en les considérant dans le plan $\left[\operatorname{Ln}\left(C_{\mathrm{g}}\right), \operatorname{Ln}(N)\right]$, où $C_{\mathrm{g}}$ est la circonférence quadratique moyenne et $N$ le nombre de tiges par hectare. L'enveloppe supérieure des valeurs possibles, dans ce plan, peut être assimilée à une droite ; on l'appelle loi d'autoéclaircie. Pour les peuplements réguliers de Chêne, cette loi a la forme suivante :

$$
\operatorname{Ln}\left(N_{\max }\right)=14-1,701 \cdot \operatorname{Ln}\left(C_{\mathrm{g}}\right)
$$

où $N_{\max }$ est le nombre maximum de tiges par ha pour un peuplement de circonférence moyenne $C_{\mathrm{g}}(\mathrm{cm})$.

À partir de cette loi, on peut construire un indice de densité comme le rapport entre le nombre de tiges d'un peuplement et le nombre de tiges maximal des peuplements de même circonférence moyenne, donné par la courbe d'autoéclaircie. L'expression en est : $R d i=\frac{N \cdot C_{\mathrm{g}}^{1,701}}{e^{14}}$, où $e$ est la base des logarithmes naturels.

\section{MODÉLISATION DES VARIATIONS DE LA FORME EN FONCTION DE DESCRIPTEURS SIMPLES DE L'ARBRE}

Nous cherchons à explorer comment l'équation de forme (1) varie lorsque nous considérons des arbres placés dans des conditions très variables :

- selon le stade de développement, en considérant des peuplements d'âges très différents ;

- selon la densité du peuplement, affectée par des régimes d'éclaircie d'intensité variable ;

- selon le degré de robustesse de l'arbre, avec plusieurs modalités : arbres malingres, correspondant aux dominés de futaie régulière, dominants plus robustes, arbres très trapus de taillis-sous-futaie.

Pour répondre à cette question, le matériel expérimental constitué par les 82 Chênes de l'échantillon INRAONF s'est révélé trop limité. En effet, il ne comprend qu'un petit nombre d'arbres, tous au stade adulte. Si les taillis-sous-futaie sont bien représentés, il y manque la gamme des sylvicultures possibles en futaie régulière et les stades juvéniles. Nous avons donc constitué le second échantillon afin de valider la démarche.

La méthode adoptée pour étudier les variations de forme entre arbres est la suivante. Dans un premier temps, nous avons scindé en deux parties l'échantillon de fiches de cubage : une partie dite «Vieux peuplements », comprenant les lots de fiches de cubage de Sablonnières Rouges et Launay-Morel (Bellême), Allées de Blois, Charmaie (Blois), Clé des Fossés, Richebourg et Morat (Tronçais), soit 754 fiches au total ; une partie dite «Jeunes peuplements», comprenant tout le reste soit 2194 fiches. Sur le lot «Vieux peuplements», nous avons ajusté le modèle [1] pour chaque arbre. Ensuite, nous avons analysé la variabilité des 7 paramètres $\mu_{i}$ et cherché à la relier à des descripteurs simples de l'arbre : âge, hauteur totale, diamètre à $1,30 \mathrm{~m}$. À cette étape, nous avons obtenu un jeu d'équations pour prédire ces paramètres. Pour finir, nous avons évalué l'ensemble de la méthode (modèle (1) et équations de prédiction des paramètres) en l'appliquant séparément à l'ensemble des données (Jeunes et Vieux peuplements) et en testant les erreurs de prévision du volume bois-fort tige.

L'ajustement de l'équation (1) a été possible pour 742 arbres sur 754, ce qui constitue un taux de réussite remarquable. Systématiquement, le paramètre $\mu_{5}$ (qui mesure l'importance du saut brutal de diamètre à la base du houppier) était fixé à une valeur appropriée pour chaque arbre. Ce choix se basait sur l'observation graphique de la qualité de l'ajustement. Pour les 6 autres paramètres, nous avons cherché à les estimer statistiquement aussi souvent que possible. Cela a été possible dans tous les cas pour les paramètres $\mu_{1}$ et $\mu_{3}$ (resp. hauteur totale de l'arbre et défilement de la tige dans le houppier). Le paramètre $\mu_{2}$ (estimation de la base du houppier) a été fixé dans 4 cas difficiles, le paramètre $\mu_{4}$ (défilement de la tige nette de branches) dans 12 cas. Les deux paramètres caractérisant l'empattement basal ont été les plus délicats : $\mu_{6}$ (empattement en diamètre à la base) n'a été estimé que dans 257 cas, $\mu_{7}$ (portée verticale de l'empattement) dans 488 cas. Lorsque l'estimation d'un paramètre était impossible ou trop imprécise, celuici était fixé à une valeur qui, graphiquement, semblait appropriée. 
Tableau I. Pourcentages des valeurs du paramètre $\mu_{5}$ par placette.

\begin{tabular}{|c|c|c|c|c|c|c|c|c|}
\hline \multirow[b]{2}{*}{ Placette } & \multirow[b]{2}{*}{ âge moyen } & \multicolumn{7}{|c|}{ Valeur de $\mu_{5}$ (saut en diamètre à la base du houppier) : } \\
\hline & & 1 & 1,1 & 1,2 & 1,3 & 1,4 & 1,5 & 1,6 \\
\hline Sablonnières rouges & 139 & 96 & 0 & 4 & 0 & 0 & 0 & 0 \\
\hline Launay Morel & 219 & 89 & 0 & 3 & 5 & 3 & 0 & 0 \\
\hline Allées de Blois & 144 & 93 & 1 & 2 & 2 & 2 & 0 & 0 \\
\hline Charmaie & 190 & 85 & 5 & 3 & 2 & 5 & 0 & 0 \\
\hline Clé des Fossés & 133 & 93 & 1 & 4 & 2 & 0 & 0 & 0 \\
\hline Richebourg & 152 & 83 & 1 & 8 & 6 & 1 & 0 & 1 \\
\hline Morat & 220 & 74 & 2 & 8 & 7 & 7 & 1 & 1 \\
\hline Total & 171 & 87,7 & 1 & 4,6 & 3,6 & 2,4 & 0,1 & 0,3 \\
\hline
\end{tabular}

\subsection{Paramètres caractérisant le profil dans le houppier}

Considérons d'abord le paramètre $\mu_{5}$ (saut de diamètre à la base du houppier). Lorsqu'il vaut 1 , il n'y a pas de saut ; autrement dit, le profil de tige est continu de bas en haut de l'arbre. Lorsqu'une discontinuité importante existe, son importance est mesurée par des valeurs supérieures à $1\left(\mu_{5}\right.$ est le rapport entre diamètres en-dessous et au-dessus de la discontinuité). Le tableau I résume la distribution de $\mu_{5}$ entre les 742 arbres. Dans presque $90 \%$ des cas, on peut retenir une valeur de 1 , et ceci quel que soit l'âge du peuplement. On se trouve pourtant dans une gamme d'âges où les cimes sont moins pyramidales et hiérarchisées, avec une fréquence de fourches et grosses branches assez élevée. Or nous avions montré sur l'échantillon INRA-ONF que l'apparition de valeurs supérieures à 1 coïncidait en général avec ces évènements, avec une fréquence générale de $1 / 3$ en futaie, 3/4 en TSF [8]. Les résultats du tableau I ne signifient pas qu'il y a très peu de fourches en futaie régulière, mais que leur présence éventuelle ne modifie pas trop brutalement le diamètre local de la tige, dans la plupart des cas. On peut donc retenir comme règle générale que le défilement des arbres de futaie est sans discontinuité majeure ; cette règle s'applique à des arbres de futaie menée assez serrée, mais elle est probablement prise en défaut dès que le traitement s'intensifie, a fortio$r i$ en TSF.

À partir de maintenant, nous travaillerons sur le souséchantillon de 644 arbres pour lesquels la hauteur totale de l'arbre est disponible (pour les autres, seule la hauteur du bois-fort était relevée). Le paramètre $\mu_{1}$, défini comme le niveau où le diamètre de la tige s'annulle, ne diffère pas significativement de la hauteur sur notre échantillon. L'écart-type des différences entre ces deux quantités s'élève à $1,22 \mathrm{~m}$. On peut donc retenir l'équation suivante, particulièrement simple :

$$
\mu_{1}=h
$$

domaine d'ajustement : hauteur $h$ comprise entre 20 et $40 \mathrm{~m}$.

Dans les travaux sur l'échantillon INRA-ONF, nous avions montré que le paramètre $\mu_{2}$, niveau de jonction entre les deux troncs de cône, ne diffère pas significativement de la base du houppier mesurée, c'est-à-dire le niveau d'insertion de la première branche vivante dont les feuilles participent à l'étage principal de végétation. Sur l'échantillon de fiches de cubage, nous ne pouvons pas vérifier ce résultat, puisque la base du houppier n'a pas été mesurée. Nous admettrons qu'il s'applique encore. Nous avons cherché à modéliser ce paramètre en considérant non pas le niveau brut, mais la longueur relative du houppier qui en est dérivée. Nous la notons $C R=1-\frac{\mu_{2}}{h}$ (en anglais, sa dénomination usuelle est Crown Ratio). CR peut être reliée très simplement, sur l'échantillon de 644 arbres, à l'accroissement radial moyen de l'arbre depuis l'origine (cette valeur, obtenue à partir du diamètre sur écorce, n'est pas confondue avec la largeur moyenne des cernes).

$$
C R=0,21424+0,14755 . i r \_m o y
$$

où ir_moy est l'accroissement radial moyen (en mm) :

$$
i r \_m o y=\frac{5 \cdot d_{130}[\mathrm{~cm}]}{a g e[\mathrm{ans}]}
$$

domaine d'ajustement : ir_moy compris entre 0,75 et $2,5 \mathrm{~mm}$.

L'équation (10) explique $15,3 \%$ de la variance de $C R$. Si nous revenons aux valeurs brutes, l'expression $h$. (1 $\left.0,21424-0,14755 . i r \_m o y\right)$ explique $22 \%$ de la variance de $\mu_{2}$, avec un écart-type résiduel de 3,6 m. On pourra s'étonner de ces faibles pourcentages d'explication. 
C'est un caractère général, qu'on retrouve quel que soit le jeu de données considéré. Nous pensons qu'il reflète le caractère fortement variable de la base du houppier chez les feuillus, lié aux différences de port entre arbres (fourches basses, faisceaux de grosses branches, cimes bien hiérarchisées avec branches horizontales se rencontrent dans tous les peuplements). Dans une autre étude non encore publiée, nous avons montré que la base du feuillage, par contre, est un caractère beaucoup moins variable entre arbres. On peut donc faire l'hypothèse que la base du feuillage est fortement influencée par la disponibilité en lumière, tandis que le niveau d'insertion des branches-support reflète en outre la variabilité du port architectural.

Pour terminer sur la base du houppier, signalons qu'un modèle analogue peut être ajusté sur l'échantillon INRA-ONF, avec des paramètres très voisins. Cela signifie que l'équation (10), malgré la forte variation résiduelle, est assez robuste et peut probablement être appliquée aussi à des arbres de taillis-sous-futaie. Enfin, le modèle signifie que, plus la croissance radiale est rapide, plus le houppier est long en proportion de la hauteur totale. Logiquement, en réalité, il faudrait renverser la proposition précédente : plus la compétition est faible, plus le houppier est long et productif, donc plus la croissance radiale est forte.

\subsection{Paramètres décrivant la partie commerciale de la tige}

Les 3 paramètres $\mu_{4}$ (défilement de la tige nette de branches), $\mu_{6}$ (importance de l'empattement en diamètre au sol) et $\mu_{7}$ (portée verticale de l'empattement) sont ceux qui ont le plus d'impact pour les applications forestières du modèle de profil de tige. En effet, ils déterminent la variation de la largeur des cernes dans les premiers mètres de la grume, sa conicité et contribuent pour une très large part au calcul du volume.

Pour rendre compte de la variabilité entre arbres de ces 3 paramètres, la variable qui s'est révélée systématiquement la plus explicative est un indice de robustesse construit à partir du diamètre et de la hauteur :

$$
d / h^{\prime}=\frac{d_{130}}{h-1,30} .
$$

Les deux paramètres définissant l'empattement sont liés à cet indice par les relations (11) et (12) :

$$
\begin{aligned}
& \mu_{6}=0,11651+0,19097 \cdot d / h^{\prime} \\
& \mu_{7}=0,28439+1,2284 . d / h^{\prime}
\end{aligned}
$$

où $d / h^{\prime}$ est l'indice de robustesse $(\mathrm{en} \mathrm{cm} / \mathrm{m})$ : domaine d'ajustement 0,75 à $3,5 \mathrm{~cm} / \mathrm{m}$.

Concernant le défilement de la tige $\mu_{4}$, nous avons pu utiliser le même prédicteur. Nous avons utilisé les 728 valeurs ajustées telles que $\mu_{4}>0,2$. La figure 2-gauche montre que la variabilité des résidus autour du modèle augmente avec l'indice de robustesse. Pour résoudre ce problème, nous avons transformé les deux variables $\left(\mu_{4}, d / h^{\prime}\right)$ en considérant leurs inverses. Cette transformation permet d'obtenir une variance homogène, plus conforme aux hypothèses de la régression linéaire (figure 2-droite). Nous avons donc ajusté l'équation suivante :

$$
\frac{1}{\mu_{4}}=0,56822+1,1758 \cdot \frac{1}{d / h^{\prime}} .
$$

Les statistiques d'ajustement des modèles (11) à (13) sont indiquées dans le tableau II. Les 3 régressions sont hautement significatives (la valeur du test-F est très forte). Pourtant, seule une faible proportion de la variance totale est expliquée : $14 \%$ pour $\mu_{6}$ et $\mu_{7}, 28 \%$ pour $\mu_{4}$. Concernant l'empattement, le même constat avait été
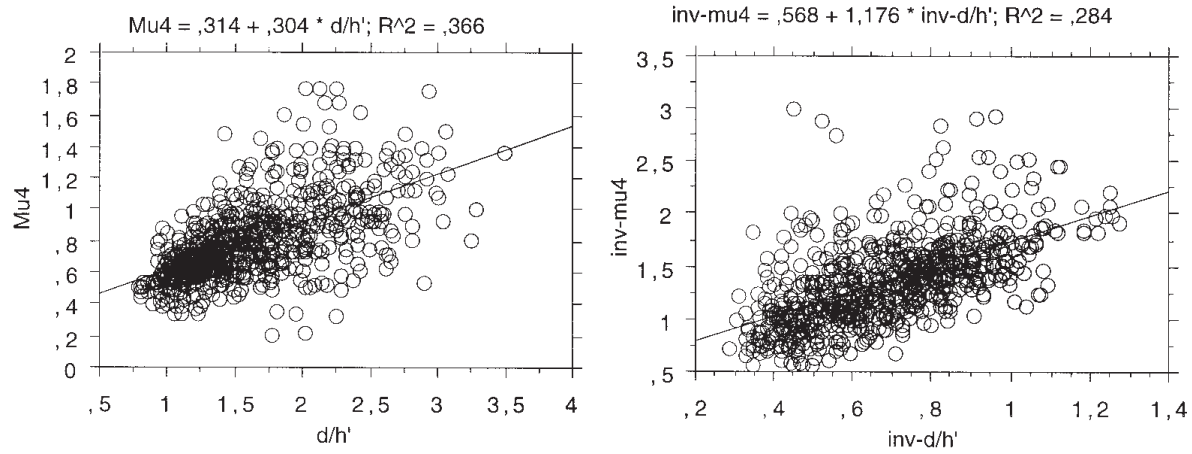

Figure 2. Régressions linéaires entre le défilement de la tige $\left(\mu_{4}\right.$ en $\mathrm{cm} / \mathrm{m}$ ) et l'indice de robustesse de l'arbre $(d / h$ ' en $\mathrm{cm} / \mathrm{m})$. À gauche : relation entre les variables brutes, à droite : relation entre les inverses des variables. 
Tableau II. Statistiques générales d'ajustement des modèles (11) à (14) (voir définitions dans le texte).

\begin{tabular}{lcccc}
\hline Equation & Effectif N & Test F de la régression & Ecart-type des résidus & $R^{2}$ \\
\hline$[11]$ & 644 & 104,2 & $0,217($ ss unité) & 0,138 \\
{$[12]$} & 644 & 109,1 & $1,37(\mathrm{~m})$ & 0,144 \\
{$[13]$} & 728 & 287,9 & $0,371(\mathrm{~m} / \mathrm{cm})$ & 0,283 \\
{$[14]$} & 728 & 220,9 & $0,346(\mathrm{~m} / \mathrm{cm})$ & 0,377 \\
\hline
\end{tabular}

fait pour les 82 Chênes de l'échantillon INRA-ONF. Deux explications peuvent être proposées à ce sujet. D'une part, l'estimation des paramètres influant sur l'empattement est difficile, parce que quelques points expérimentaux seulement sont concernés (les 2 à 4 premiers niveaux de mesure). Les paramètres sont donc entachés d'une erreur d'estimation relativement forte. D'autre part, l'intensité (en diamètre) et la portée verticale de l'empattement sont probablement des caractères très variables entre arbres, même dans un peuplement donné. Quant au défilement de la tige, nous obtenons un pourcentage d'explication meilleur, quoique loin d'être excellent. Nous allons voir qu'on peut améliorer la prévision de ce défilement en considérant la position de la base du houppier.

\subsection{Influence de la longueur du houppier sur la forme de la grume}

Dans le sous-échantillon des 742 vieux Chênes, la hauteur de la base du houppier estimée grâce au modèle de profil de tige varie entre 5 et $30 \mathrm{~m}$ (moyenne 18,4 m, écart-type 3,98 m). La longueur relative du houppier $C R$, quant à elle, varie entre 0,10 et 0,86 (moyenne 0,413 , écart-type 0,121). Cette gamme de morphologies est très large et recouvre bien l'ensemble des valeurs observables en futaie au stade adulte. Les valeurs maximales pour $C R$ sont analogues à celles qu'on peut obtenir pour des Chênes de taillis-sous-futaie.

Nous avons constaté qu'on pouvait améliorer très nettement la prédiction du défilement de la tige en considérant simultanément deux variables indépendantes : l'indice de robustesse et la longueur relative du houppier. Pour cela, nous procédons par régression multiple. On doit au préalable s'assurer que ces deux prédicteurs sont bien indépendants : sur notre échantillon, le coefficient de corrélation simple entre eux est de 0,28 , une valeur suffisamment faible pour permettre d'estimer la contribution respective des deux variables avec une bonne fiabilité. Bien sûr, toutes les combinaisons de ces deux grandeurs ne sont pas possibles in natura. Les arbres à houppier court $(C R=0,2)$ ont des robustesses comprises entre 0,75 et $2 \mathrm{~cm} / \mathrm{m}$; lorsque le houppier représente la moitié de la hauteur totale, les robustesses possibles vont de 0,9 à $3 \mathrm{~cm} / \mathrm{m}$. Finalement, il manque dans notre échantillon toutes les morphologies d'arbres de taillis-sous-futaie : robustesse comprise entre 2,5 et $5 \mathrm{~cm} / \mathrm{m}$, houppier s'étendant sur 50 à $80 \%$ de la hauteur. Le modèle suivant a été ajusté :

$$
\frac{1}{\mu_{4}}=1,3443 \cdot \frac{1}{d / h^{\prime}}+1,1021 \cdot C R .
$$

Par rapport à [13], on constate (tableau II) que la proportion de variance expliquée augmente de 28 à $38 \%$. Le sens des effets est intéressant : comme précédemment, plus l'arbre est robuste, plus son défilement est fort ; mais, pour un couple (hauteur, diamètre) donné, un houppier plus long entraine un défilement plus faible, c'est-à-dire une grume plus cylindrique. Pour apprécier l'intensité de ces effets, nous avons comparé à la figure 3 les prévisions des modèles (13) et (14), en considérant dans ce deuxième cas des longueurs relatives de houppier variant de 10 à $80 \%$. Les deux modèles sont très

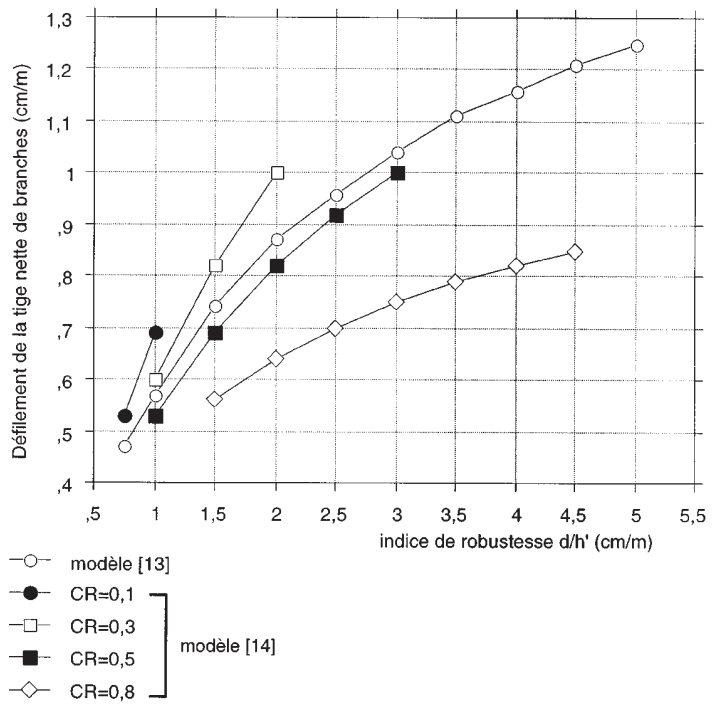

Figure 3. Prévision de défilement de la tige en fonction de l'indice de robustesse de l'arbre, par les deux modèles (13) et (14). Pour le modèle (14), les résultats sont fournis pour 4 valeurs de $C R$ (longueur relative du houppier). Seules les combinaisons $\left[d / h^{\prime}, C R\right]$ réalistes sont représentées. 
proches l'un de l'autre pour des houppiers représentant environ $50 \%$ de la hauteur (c'est logique, puisqu'il s'agit là du point «moyen» dans notre échantillon). Par contre, le modèle (14) permet de prévoir une grande diversité de forme des grumes, à diamètre et hauteur fixés. Des arbres à houppier très long, de type TSF, ont une grume nettement moins conique que des sujets élancés, ce qui confirme bien l'impression visuelle ressentie lorsqu'on ajuste les profils de tige un par un.

Nous pouvons tirer des conclusions importantes de cette analyse. D'abord, si l'on veut estimer précisément la forme de la grume (conicité-cylindricité), il est fondamental de connaître avec précision non seulement le diamètre et la hauteur de l'arbre, mais encore la position de la base du houppier. Or, dans la présente étude, nous avons indiqué que la seule connaissance d'un triplet (âge, diamètre, hauteur) ne permet d'expliquer que $15 \%$ de la variabilité de la longueur relative du houppier. Par conséquent, il subsiste une très forte variation entre arbres, intra-peuplement, dont nous venons de constater l'effet important sur la forme. Si l'on considère l'intérêt actuel pour une bonne prédiction des relations sylviculture-croissance-qualité [13] pour l'Epicéa commun [17] (pour le Chêne), il est donc indispensable de pouvoir simuler la dynamique de la base du houppier ; et ceci sans se contenter de la moyenne par peuplement ou par classes de diamètre, mais en abordant concrètement les variations entre arbres. Cela nécessite de s'appuyer sur une bonne connaissance botanique du développement aérien des feuillus et de l'intégrer, même sous forme simplifiée, dans des modèles de croissance opérant à différentes échelles (simulateurs du développement architectural, modèles d'arbre dendrométriques dépendants ou indépendants des distances). Les recherches menées actuellement par les équipes INRA-Croissance de Nancy-Champenoux, CIRAD-INRA AMAP de Montpellier, l'Office National des Forêts et la Forestry Commission, dans le cadre du Projet Européen OAKKEY, vont apporter les éléments nécessaires pour le Chêne sessile [6].

Avant de poursuivre dans les applications numériques, on peut discuter brièvement la question de savoir pourquoi les Chênes à houppier long ont un tronc plus cylindrique que ceux à houppier court. Nos analyses précédentes $[11,12]$ avaient montré que, chez le Chêne sessile, la répartition longitudinale des cernes annuels n'obéit pas à la loi de Pressler (1865) : en effet, ce n'est pas leur surface, mais leur épaisseur qui est conservée sous le houppier. De plus, nous avions noté que les cernes étaient généralement un peu plus larges à l'intérieur du houppier. La conjonction de ces deux caractères permet de comprendre pourquoi la tige des Chênes peut être raisonnablement assimilée à l'assemblage de deux troncs de cône (l'ajout d'une couche annuelle de bois de largeur uniforme perpétue la forme conique). De façon plus locale, nous avions relevé [11] que, sur de courtes longueurs vers le bas du houppier, la surface des cernes était uniforme. Ainsi, la loi de Pressler ne s'appliquerait que très localement : voici comment on passe de cernes larges et fort défilement, dans le houppier, à des cernes étroits et faible défilement, le long de la tige nette de branches. Ces constats morphologiques nous ont conduit à poser la question du rôle des branches vis-à-vis de la tige : sont-elles autonomes, exportatrices ou importatrices nettes d'assimilats ; comment cette activité se modifie-t-elle du haut en bas du houppier; quelles règles régissent les accroissements au voisinage d'une ramification; quel en est l'impact sur l'acquisition de la forme de la tige ? Ces questions ont été partiellement abordées par I. Planchais pour le jeune Hêtre (1998) et sont actuellement approfondies par Stéphanie Pouderoux, dans sa thèse consacrée au Hêtre et au Chêne sessile aux stades perchis-jeune futaie. Nous estimons que ces recherches vont permettre d'éclairer l'éventuel effet bénéfique des houppiers longs sur la forme de la tige, suggéré par la présente analyse.

\subsection{Estimation du volume bois-fort total (branches comprises)}

Le volume bois-fort total est une grandeur essentielle pour qui s'intéresse à la productivité primaire des écosystèmes. Posséder une bonne méthode d'estimation est aussi important en pratique, afin de séparer rigoureusement le volume commercialement utile du volume de houppier. Ce dernier point revêt une importance particulière chez les Chênes, dont une bonne partie de la ressource est composée de taillis-sous-futaie (réserves très branchues).

Dans l'échantillon des 2948 fiches de cubage, nous n'avons pas saisi le volume bois-fort total des arbres, l'objectif étant de valider l'approche profil de tige. Nous avons donc basé notre étude sur le seul échantillon INRA-ONF, qui ne comporte que 82 arbres. Sur ces données, nous avons constaté que le tarif bois-fort total de J. Bouchon estimait correctement le volume des arbres de futaie (sous-estimation moyenne de 2,4\%, écart-type des écarts $9 \%$, erreurs extrêmes -14 et $+28 \%$ ), mais par contre sous-estimait celui des réserves de taillis-sousfutaie de $13 \%$ en moyenne (écart-type de $14,5 \%$, erreurs extrêmes -8 à $+55 \%$ ). Nous avons donc construit une méthode alternative. Sur l'échantillon INRA-ONF, le rapport entre volume bois-fort total observé et volume prédit par le profil de tige augmente linéairement avec l'indice de robustesse de l'arbre, selon l'équation suivante (écart-type résiduel $=0,217, R^{2}=0,651$ pour 81 arbres) :

$$
\frac{v b f \_t o t \_o b s e r v e ́}{v b f \_t i g e \_p r o f i l}=0,563+0,375 \cdot \frac{d_{130}}{h-1,3} \text {. }
$$


Les arbres de TSF sont dans le prolongement exact de ceux de futaie, dans le plan défini par ce modèle. Si nous estimons le volume total par cette équation, nous surestimons de $1,1 \%$ les arbres de futaie et nous sous-estimons de 1,4\% ceux de TSF. L'écart-type des écarts est ramené à $11,8 \%$ en TSF. Cette méthode est donc très peu biaisée et de faible variance, par comparaison avec le tarif Bouchon. Il serait maintenant indiqué de chercher à la valider sur quelques centaines d'arbres, en privilégiant la comparaison futaie-TSF (les données en grand nombre dont nous disposons actuellement concernent exclusivement la futaie). Cette formule signifie que, plus l'arbre est robuste, plus forte est la proportion du houppier dans le volume total. Pour une application à des arbres quelconques, nous censurons la valeur estimée en imposant un minimum de 1 (le volume total doit être supérieur ou égal au volume de tige).

\section{VALIDATION DE LA MÉTHODE ET COMPARAISON AVEC D'AUTRES SOURCES}

Le modèle de profil de tige (1), associé aux équations (9) à (13), permet de prédire la forme de n'importe quel arbre dès que l'on connaît son triplet (âge, diamètre, hauteur). Notons que nous ne considérons pas l'équation (14), puisque nous n'avons pas d'information précise sur la base du houppier ; par ailleurs, il n'est pas nécessaire de disposer d'une équation pour le défilement de la tige dans le houppier, puisque ce dernier se déduit de tout le reste (la courbe de profil est astreinte à passer par le diamètre de l'arbre au niveau $1,30 \mathrm{~m}$ ). A partir de la courbe de profil, il est possible de calculer le volume de la tige à une découpe quelconque.

\subsection{Erreur de prévision sur le volume bois-fort tige}

Nous utiliserons comme critère de validation le volume de tige à la découpe bois-fort (diamètre $7 \mathrm{~cm}$ ). Nous chercherons à estimer les erreurs de prévision de ce volume à partir du profil (biais et variance résiduelle). La procédure a été appliquée à l'échantillon de 2948 fiches de cubage ainsi qu'à l'échantillon INRA-ONF. Le pre- mier sera utilisé ici en distinguant «Vieux peuplements» (sous-échantillon de construction de la méthode) et «Jeunes peuplements» (validation pure). Classiquement, on observe que la variance du volume augmente avec le volume (hétéroscédasticité). Nous avons donc utilisé comme critère d'analyse le rapport entre le volume observé $(v b f t)$ et le volume estimé par le profil, noté $v b f t p$. Nous avons également considéré, pour apprécier l'apport de la méthode profil, le tarif de cubage à deux entrées établi par [4], noté $v b f t J B$.

L'examen des erreurs d'estimation sur le volume montre d'abord que les 332 arbres situés juste au-dessus de la limite bois-fort (circonférences comprises entre 20 et $30 \mathrm{~cm}$ ) sont régulièrement sous-estimés : le ratio entre volumes observés et prédits varie de 1 à 3,3. Pour les 2616 arbres restants (voir figure 4), les erreurs sont distribuées de façon parfaitement normale ; le biais moyen est de $2 \%$ pour les Vieux peuplements (échantillon de construction), de $3 \%$ pour les Jeunes peuplements (échantillon de validation) ; l'écart-type est réduit et représente 8 à $10 \%$ de la valeur prédite. Lorsqu'on cherche à relier l'erreur de prédiction à diverses variables caractéristiques de l'arbre, on trouve la plus forte corrélation simple avec l'accroissement radial moyen (corrélation de -0,145). Le volume est sous-estimé de 5,6\% lorsque l'accroissement radial est de $0,5 \mathrm{~mm}$, surestimé de $3,9 \%$ pour $3 \mathrm{~mm}$. Globalement, on peut donc affirmer que la méthode est correcte, pour le critère du volume qui a été retenu.

On peut maintenant considérer ensemble et comparer l'estimation du volume par deux méthodes : 1) à partir du profil de tige simulé ; 2) grâce au tarif de cubage bois-fort tige de [4]. En travaillant sur l'ensemble des données de circonférence supérieure à $30 \mathrm{~cm}$ (tableau III), on constate que les deux méthodes conduisent à des biais assez réduits (resp. sous-estimations de 2,7 et $1,5 \%$ ) ; l'écart-type des erreurs est un peu plus élevé avec le tarif de J. Bouchon. Les erreurs du tarif J. Bouchon sont un peu plus fortes (surestimations) pour les gros diamètres et les vitesses de croissance fortes. Quant aux arbres de circonférence comprise entre 20 et $30 \mathrm{~cm}$, les deux méthodes ont des performances inverses : la méthode profil de tige sous-estime nettement, tandis que le tarif surestime. Dans les 2 cas, on peut donc conclure que d'une part, l'estimation du

Tableau III. Comparaison entre les erreurs d'estimation multiplicatives pour le volume : d'après la méthode du profil de tige et d'après le tarif de cubage de J. Bouchon.

\begin{tabular}{lcccccc}
\hline & Moy. & Dév. Std & Erreur Std & Nombre & Minimum & Maximum \\
\hline vbft (obs/profil) & 1,027 & 0,088 & 0,002 & 2616 & 0,587 & 1,526 \\
vbft (obs/tarif_JB) & 1,015 & 0,099 & 0,002 & 2616 & 0,590 & 1,546 \\
\hline
\end{tabular}




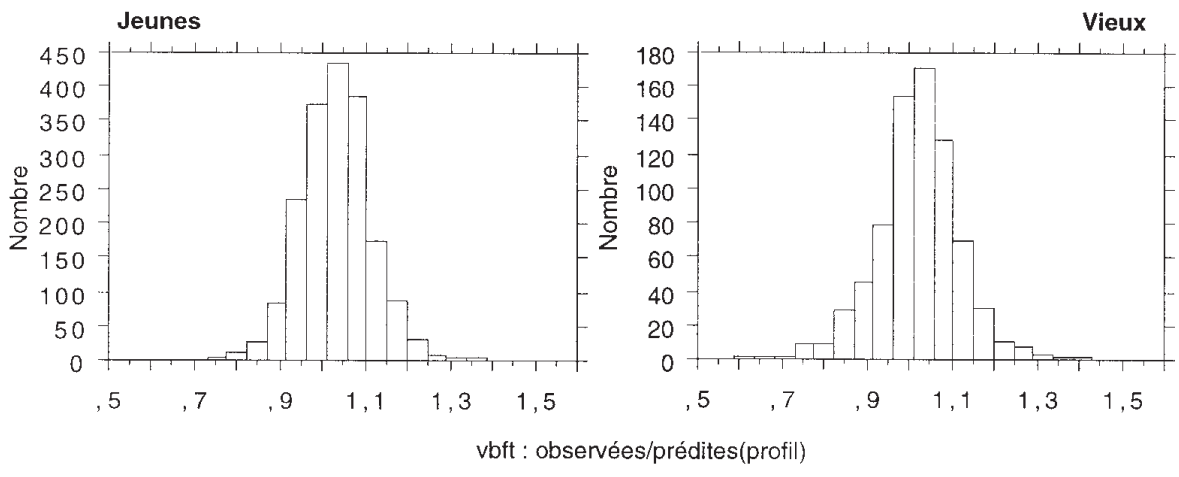

Statistiques descriptives

\begin{tabular}{|c|c|c|c|c|c|c|}
\hline \multirow{4}{*}{$\begin{array}{ll}\text { vbft(obs/profil), } & \text { Total } \\
\text { vbft(obs/profil), Jeunes } \\
\text { vbft(obs/profil), } & \text { Vieux }\end{array}$} & Moy. & Dév. Std & Erreur Std & Nombre & Minimum & Maximum \\
\hline & 1,027 & .088 &, 002 & 2616 & .587 & 1,526 \\
\hline & 1,030 &, 083 &, 002 & 1862 & .661 & 1,526 \\
\hline & 1,020 &, 100 & .004 & 754 &, 587 & 1,425 \\
\hline
\end{tabular}

Figure 4. Statistiques de l'erreur multiplicative sur le volume bois-fort tige lorsqu'on l'estime grâce au profil de tige (Eqs. (1) et (9) à (13)). Seuls les arbres de circonférence supérieure à $30 \mathrm{~cm}$ sont représentés. volume des très jeunes arbres doit être améliorée, d'autre part les deux méthodes sont suffisamment cohérentes et précises dans la gamme de diamètres 10 à $100 \mathrm{~cm}$.

Nous avons analysé les erreurs de prévision du volume par la méthode profil de tige, en les subdivisant par placette. L'échantillon des 2616 arbres de circonférence supérieure à $30 \mathrm{~cm}$ a été considéré. Il est réparti entre 22 placettes appartenant à 4 forêts ; l'âge moyen par placette s'étend de 70 à 220 ans ; l'indice de densité de peuplement $R d i$ (dont les valeurs théoriques sont comprises entre 0 et 1) diffère également entre placettes, ses valeurs moyennes par placette sur toute la durée d'observation s'étalant entre 0,524 (Ducellier) et 0,948 (Trésor 1). Ces placettes couvrent donc bien le domaine de la futaie régulière entre les stades perchis et futaie adulte, ainsi que les différents itinéraires sylvicoles possibles (à l'exception des sylvicultures très énergiques).

Nous avons cherché à savoir si les volumes étaient significativement biaisés par placette. La variable utilisée est le rapport entre volumes observé et prédit ; nous avons comparé, par un test-t univarié, cet indice à la valeur théorique 1,027 , qui est la moyenne générale de tout l'échantillon. Cela revient donc à tester si telle placette est plus ou moins biaisée que l'ensemble. 7 placettes sur 22 ont des écarts différents en moyenne de 1,027. Les valeurs moyennes extrêmes sont de 0,955 (Charmaie) et 1,092 (Sablonnières 2B). Il n'apparaît aucune relation entre l'intensité du biais et l'âge ou la densité moyenne de la placette.

Pour en terminer avec la validation, nous avons également comparé la performance des deux méthodes de cubage pour l'échantillon INRA-ONF, en le subdivisant en futaie et TSF. Sur ces données, le tarif de J. Bouchon surestime le volume bois-fort tige de $6 \%$ en futaie, de $24 \%$ en TSF : ce dernier biais est considérable mais doit être nuancé par le fait que ce tarif était construit pour (et grâce à) des arbres de futaie. La méthode basée sur le profil de tige présente des biais plus réduits : $-0,2 \%$ en futaie, $+6 \%$ en TSF. Pour les deux méthodes, l'écarttype des écarts s'élève à $7 \%$ en futaie, $10 \%$ en TSF, en valeurs relatives.

En conclusion, on peut donc affirmer que la méthode basée sur le profil de tige est particulièrement robuste vis-à-vis de l'âge, de la densité des peuplements (y compris celle des taillis-sous-futaie) et de la position géographique. Seuls les diamètres inférieurs à $10 \mathrm{~cm}$ sont mal estimés. Avec la même restriction relative aux très petits diamètres, on pourra aussi utiliser le tarif de J. Bouchon pour les arbres de futaie, les deux méthodes donnant des résultats très similaires. La méthode-profil de tige nécessite, en entrée, le diamètre, la hauteur et l'âge de l'arbre ; de plus, les calculs sont assez complexes. Ce n'est donc pas, a priori, une solution à proposer pour un cubage rapide de peuplements en gestion courante. Par contre, c'est une méthode souple, appropriée pour être intégrée dans des simulateurs de la croissance.

\subsection{Comparaison de tarifs de cubage «recherche» et «aménagement»}

À plusieurs reprises, au cours des dernières années, les prévisions de volume (stock sur pied et accroissement courant) fournies par notre modèle Chêne ont été jugées trop fortes par nos partenaires de l'Office National des Forêts qui l'ont utilisé. Le premier cas de désaccord 
entre chiffres « recherche» et «gestion» concernait d'abord une chênaie du Plateau lorrain, site pilote pour la gestion des oiseaux et divers animaux : nos prévisions avaient été rejetées car beaucoup plus fortes que celles de la table de production de [20]. Nous reviendrons plus loin sur la comparaison de nos estimations avec cette table. Le second cas visait, plus largement, les chênaies de Basse-Normandie [1] : le volume sur pied en fin de révolution prédit par le simulateur s'élevait à environ $700 \mathrm{~m}^{3} / \mathrm{ha}$, alors que les services locaux travaillaient plutôt sur une base de $500 \mathrm{~m}^{3} / \mathrm{ha}$, pour des peuplements gérés de façon similaire.

Plusieurs causes peuvent être invoquées pour expliquer ces désaccords. La plus évidente est une différence entre les objets considérés (volume tige seule ou volume total). On peut en général, si les méthodes sont bien renseignées, écarter ce problème en choisissant le tarif «recherche» correspondant au volume cubé par le tarif «gestion». La seconde explication a trait aux problèmes de surface : les volumes calculés en gestion courante sont relatifs à une parcelle entière (10 à 30 ha), alors que ceux des placettes des Instituts de recherche concernent des surfaces plus faibles. Les parcelles entières supportent en principe des vides, trouées de chablis, emprises de routes, qui doivent conduire à des réfactions. De plus, en raison des effets de bord, les placettes de petite surface peuvent supporter un matériel sur pied très élevé, sans rapport avec la parcelle entière. Ce second argument s'applique, nous semble-t-il, aux placettes de quelques ares, mais pas à nos propres données (placettes de 1 à 2 ha, exceptionnellement 0,5 ha). Le dernier argument a trait à l'inadéquation des tarifs de cubage (différences de forme mal prises en compte, difficulté à estimer le volume du houppier). Nous avons cherché à évaluer ce dernier argument sur un cas particulier, le tarif Aménagement de Tronçais, renseigné dans le Plan de Gestion (Aménagement de Tronçais, [19], p. G.2.1).

D'après les termes mêmes du Plan de Gestion, le tarif Aménagement fournit «une approximation du volume total tige plus houppier». Il s'agit d'un tarif à une entrée (tableau $I V$ ), que nous pouvons comparer au tarif à deux entrées pour le volume bois-fort total (tige et branches) de J. Bouchon. Pour faire cette comparaison, nous devons estimer la hauteur moyenne par classe de diamètre pour la Forêt de Tronçais. À cette fin, nous utilisons l'ensemble des mesures disponibles dans l'échantillon de fiches de cubage, qui est bien réparti sur l'ensemble de la forêt et dans toutes les classes d'âge. La courbe que nous avons ajustée est $h=36,5$. (1 -

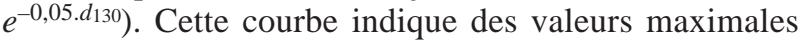
voisines de $36 \mathrm{~m}$ pour les diamètres les plus gros (canton de Morat), chiffre identique à nos mesures récentes dans la partie réservée de la Futaie Colbert. Nous pensons donc que cette courbe est une bonne description de la forêt dans son état actuel. Nous avons montré précédemment le caractère non-biaisé du tarif bois-fort total de J. Bouchon pour des Chênes représentatifs de la futaie ligérienne. Par conséquent, nos estimations de volume peuvent être considérées comme une très bonne approximation pour Tronçais.

On peut voir, au tableau IV, que le tarif Aménagement est systématiquement (quel que soit le diamètre) inférieur de $25 \%$ au tarif «recherche». Cette différence est vraiment énorme et suffirait à justifier, pour un peuplement mûr typique (100 tiges par ha de diamètre moyen $70 \mathrm{~cm}$ ) un écart de $200 \mathrm{~m}^{3} / \mathrm{ha}$ pour le volume sur pied, c'est-à-dire un écart analogue à celui mentionné en Basse-Normandie.

\subsection{Comparaison avec la Table de Production de Pardé (1962)}

Pour permettre aux lecteurs de resituer nos prévisions de volume (stock et accroissement) par rapport aux travaux précédents de [20], dont la table de production à classe de fertilité unique avait été établie à partir des mêmes données, nous comparons à la figure 5 les courbes obtenues pour la hauteur dominante et l'accroissement courant en volume de bois-fort tige. La comparaison est valide parce que la sylviculture de la Table de Pardé est analogue à celle que nous avons simulée.

S'agissant de la hauteur, on constate que la croissance prévue par la Table est presque confondue avec celle annoncée par [10], pour une bonne fertilité, jusqu'à l'âge

Tableau IV. Volume unitaire par classes de diamètre pour Tronçais, d'après le tarif aménagement et d'après le tarif à deux entrées de J. Bouchon.

\begin{tabular}{|c|c|c|c|}
\hline \multirow[b]{2}{*}{$\begin{array}{l}d_{130} \\
(\mathrm{~cm})\end{array}$} & \multicolumn{3}{|c|}{ volume bois-fort total selon } \\
\hline & $\begin{array}{l}\text { hauteur } \\
\text { (m) }\end{array}$ & $\begin{array}{l}\text { tarif aménagement } \\
\mathrm{m}^{3}\end{array}$ & $\underset{\mathrm{m}^{3}}{\operatorname{tarif} \mathrm{J} . \mathrm{B}}$ \\
\hline 20 & 23,1 & 0,3 & 0,4 \\
\hline 30 & 28,4 & 0,8 & 1,1 \\
\hline 40 & 31,6 & 1,7 & 2,2 \\
\hline 50 & 33,5 & 2,7 & 3,6 \\
\hline 60 & 34,7 & 4,0 & 5,3 \\
\hline 70 & 35,4 & 5,4 & 7,4 \\
\hline 80 & 35,8 & 7,2 & 9,7 \\
\hline 90 & 36,1 & 9,1 & 12,3 \\
\hline 100 & 36,3 & 11,3 & 15,1 \\
\hline 110 & 36,4 & 13,7 & 18,1 \\
\hline 120 & 36,4 & 16,2 & 21,3 \\
\hline
\end{tabular}



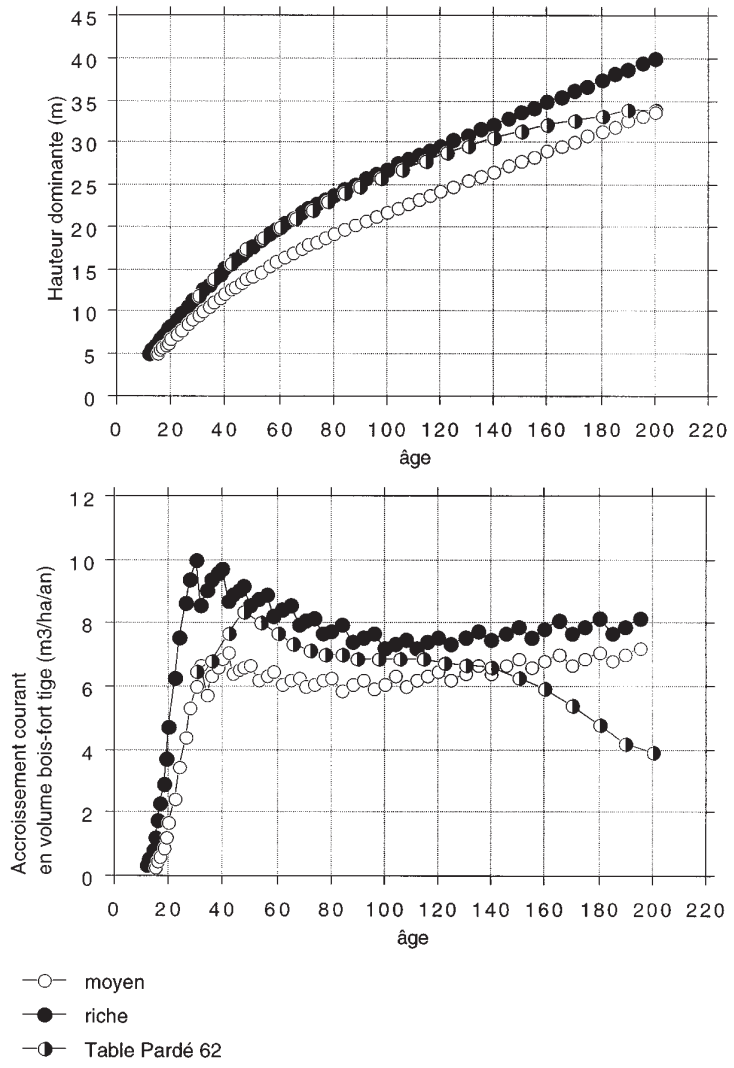

Figure 5. Comparaison entre nos simulations pour des stations riche et moyenne d'une part, d'autre part la Table de Production à classe de fertilité unique de Pardé (1962). En haut: hauteur dominante ; en bas : accroissement courant en volume bois-fort tige.

de 120 ans environ, puis s'effondre sérieusement. Cette divergence au stade adulte avait été notée et discutée par [10]. Grâce à notre modèle, nous pouvons la confirmer très exactement en ce qui concerne l'accroissement en volume.

Cette divergence s'explique d'abord par la nature des données disponibles en 1962, pour la construction de la Table : à l'époque, les peuplements de plus de 120 ans étaient peu abondants dans la base de données (nous disposons maintenant de 30 années supplémentaires d'observations). Sur la base du modèle de croissance en hauteur établi par l'ONF, nous pouvons aussi affirmer que les placettes permanentes les plus âgées sont moins fertiles que les plus jeunes : en alignant graphiquement des placettes différant par l'âge et la fertilité, comme l'avait sans doute fait J. Pardé, on biaise les tendances. Enfin, nos récentes analyses du même jeu de données ont démontré que la productivité des chênaies est en aug- mentation constante depuis 1930. Ceci explique pourquoi nos prévisions d'accroissement courant en volume restent très soutenues, sans signe de diminution, dans toute la phase adulte. Cette tendance, bien que déjà à l'œuvre, était moins apparente dans les années 1960 et ne pouvait pas être détectée.

Tous les éléments disponibles montrent donc qu'il faut maintenant cesser de se référer à la Table de Production de Pardé, qui sous-estime très fortement la production des futaies adultes.

\section{SIMULATIONS DE LA PRODUCTION DES CHÊNAIES}

Nous utilisons maintenant l'ensemble des résultats précédents, au moyen de simulations, pour étudier diverses facettes de la production en volume des chênaies. Les facteurs dont nous étudions l'influence sont d'une part la fertilité de la station, d'autre part le régime d'éclaircies.

\subsection{Quatre itinéraires sylvicoles}

Nous avons considéré 4 itinéraires sylvicoles, sous l'hypothèse d'une fertilité moyenne (hauteur : $25 \mathrm{~m}$ à 100 ans) et d'un peuplement initial issu de régénération naturelle, très dense (38000 tiges/ha à 13 ans et $5 \mathrm{~m}$ de hauteur). Les itinéraires sont définis comme suit :

- Pas de sylviculture : on ne récolte rien, sauf la production finale restant en fin de révolution ; ce régime est intéressant comme référence biologique (il fournit le stock maximal permis par la station). En termes dendrométriques, il correspond à $R d i=1$ (autoéclaircie) ;

- Classique : ce scénario correspond assez bien à la pratique actuelle d'une sylviculture prudente des chênaies; il repose sur 2 dépressages légers (à 26 et 38 ans), puis 15 coupes modérées entre 60 et 190 ans ; la rotation est de 8 ans avant 100 ans, puis 10 ans ; les éclaircies sont par le haut au départ, puis mixtes ; la densité $R d i$ reste au voisinage de 0,8 ;

- Très dynamique : ce scénario («futaie claire») est la sylviculture très dynamique avec dépressages recommandée par [14] ; 2 dépressages forts à 15 et 27 ans, puis 15 éclaircies moyennes à fortes de 40 à 165 ans (rotations identiques au précédent, éclaircies un peu plus par le haut) ; la densité Rdi reste au voisinage de 0,6;

- Quasi-TSF : ce scénario a été imaginé de manière à produire des arbres dont la croissance et la morphologie se rapprochent des réserves de taillis-sous-futaie ; il s'agit cependant d'une vraie futaie régulière, avec deux 
dépressages analogues au cas précédent, puis 6 coupes très fortes et très espacées (16 ans jusqu'à 100 ans, puis 30 ans), telles que la densité $R d i$ est ramenée à chaque éclaircie de 0,5 à 0,3 (chacune prélève donc $40 \%$ du stock sur pied).

\subsection{Indice de fertilité et production en volume}

Pour comparer entre eux des peuplements de fertilités différentes, nous avons appliqué une même sylviculture, laquelle ressemble au scénario Classique (on maintient la densité autour de 0,7). Les sylviculteurs en Chênaie pourront donc appliquer directement les résultats à leurs peuplements. Nous considérons 4 niveaux de fertilité, pour des hauteurs dominantes à 100 ans de 17, 22, 27, $32 \mathrm{~m}$, ce qui balaye complètement la gamme des possibles. Les résultats sont résumés dans le tableau $V$.

L'application d'une même sylviculture conduit à garder un nombre d'arbres supérieur dans les peuplements moins fertiles : sensiblement 2,5 fois plus sur la station la plus médiocre par rapport à la plus fertile. Précisons que, malgré cette différence, la densité biologique globale - et donc l'intensité de la compétition - sont similaires dans tous les cas de fertilité, parce que les diamètres grossissent plus vite sur bonne station. Le tableau $V$ indique, à toutes fins utiles, les caractéristiques de la population finale des 70 plus gros arbres par ha, correspondant en gros à celle des arbres d'avenir. La base du houppier est étagée en fonction de la fertilité et donne une indication des longueurs de bille élaguée qu'on peut espérer sous l'hypothèse d'une sylviculture assez prudente : de 13 à $20 \mathrm{~m}$, ce qui correspond bien à la gamme mesurée aujourd'hui dans les belles futaies régulières de Chêne.

La production en volume varie dans une large gamme, à la fois selon l'indice de fertilité et selon la définition retenue pour le volume. La production biologique brute (volume bois-fort total) double entre les plus faibles et plus fortes fertilités (de 5,2 à 10,3 $\mathrm{m}^{3} \mathrm{ha}^{-1} \mathrm{an}^{-1}$ ); le même

Tableau V. Production en volume des Chênaies simulée selon l'indice de fertilité. Sont indiqués, pour le peuplement final : hauteur dominante, nombre de tiges et surface terrière par ha, diamètre dominant, volume bois-fort total et tige seule, volume de grume jusqu'au premier défaut. Pour l'arbre moyen des 70 plus gros par ha, les mêmes variables. Les accroissements moyens en volume du peuplement de 0 ou 100 ans jusqu'à 200 ans.

\begin{tabular}{|c|c|c|c|c|c|}
\hline Variable & Unité & Très riche & $\begin{array}{c}\text { é de la s } \\
\text { Riche }\end{array}$ & Moyenne & Médiocre \\
\hline \multirow[t]{2}{*}{ Indice de fertilité : $H_{0}(100$ ans $)$} & $\mathrm{m}$ & 32 & 27 & 22 & 17 \\
\hline & \multicolumn{5}{|c|}{ Valeurs sur pied à 200 ans } \\
\hline Hauteur dominante $H_{0}$ & $\mathrm{~m}$ & 46,2 & 40 & 33,7 & 27,5 \\
\hline Nombre de tiges par ha $\mathrm{N}$ & $\mathrm{ha}^{-1}$ & 64 & 83 & 112 & 139 \\
\hline Surface terrière $G$ & $\mathrm{~m}^{2} \mathrm{ha}^{-1}$ & 38,2 & 36,5 & 35,2 & 32,0 \\
\hline Diamètre dominant $D_{0}$ & $\mathrm{~cm}$ & 87,1 & 74,8 & 64,3 & 56,1 \\
\hline Volume bois-fort total Vtot7 & $\mathrm{m}^{3} \mathrm{ha}^{-1}$ & 977 & 832 & 699 & 548 \\
\hline Volume bois-fort tige Vtige 7 & $\mathrm{~m}^{3} \mathrm{ha}^{-1}$ & 755 & 641 & 537 & 408 \\
\hline Volume de grume & $\mathrm{m}^{3} \mathrm{ha}^{-1}$ & 567 & 500 & 435 & 338 \\
\hline
\end{tabular}

Population des (70) plus gros arbres restant à 200 ans, valeurs moyennes

Nombre
Diamètre
Base du houppier $\left(=1^{\text {er }}\right.$ défaut $)$
vtot7
vtige7
vgrume

$\begin{array}{ccccc}\mathrm{ha}^{-1} & 64 & 70 & 70 & 70 \\ \mathrm{~cm} & 87,1 & 76,7 & 66,3 & 57,5 \\ \mathrm{~m} & 19,2 & 17,8 & 15,9 & 13,7 \\ \mathrm{~m}^{3} & 15,2 & 10,6 & 7 & 4,5 \\ \mathrm{~m}^{3} & 11,8 & 8,1 & 5,2 & 3,3 \\ \mathrm{~m}^{3} & 8,9 & 6,3 & 4,2 & 2,7\end{array}$

\begin{tabular}{|c|c|c|c|c|c|}
\hline \multirow{4}{*}{$\begin{array}{l}\text { Vtot7 } \\
\text { Vtige7 } \\
\text { Vgrume }\end{array}$} & \\
\hline & $\mathrm{m}^{3} \mathrm{ha}^{-1} \mathrm{an}^{-1}$ & 10,31 & 8,42 & 6,75 & 5,22 \\
\hline & $\mathrm{m}^{3} \mathrm{ha}^{-1} \mathrm{an}^{-1}$ & 8,85 & 7,21 & 5,70 & 4,28 \\
\hline & $\mathrm{m}^{3} \mathrm{ha}^{-1} \mathrm{an}^{-1}$ & 5,88 & 4,83 & 3,85 & 2,89 \\
\hline \multicolumn{6}{|c|}{ Accroissements moyens en volume de 100 à 200 ans } \\
\hline Vtot7 & $\mathrm{m}^{3} \mathrm{ha}^{-1} \mathrm{an}^{-1}$ & 11,20 & 9,70 & 8,40 & 6,92 \\
\hline Vtige7 & $\mathrm{m}^{3} \mathrm{ha}^{-1} \mathrm{an}^{-1}$ & 8,66 & 7,61 & 6,57 & 5,33 \\
\hline Vgrume & $\mathrm{m}^{3} \mathrm{ha}^{-1} \mathrm{an}^{-1}$ & 6,51 & 6,05 & 5,57 & 4,87 \\
\hline
\end{tabular}


ratio est observé pour le volume bois-fort tige et pour le volume arrêté au premier défaut, considéré ici comme la base du houppier (première branche vivante à l'exception des gourmands).

Quelle que soit la station, la production du volume commercial le mieux valorisé représente $55 \%$ seulement de la production biologique. Ce faible rendement est dû au fait que la production de volume-grume, au sens où nous l'avons défini ici (jusqu'à la découpe $20 \mathrm{~cm}$ ou au premier défaut), ne démarre vraiment qu'après 40 ans sur très bonne station, 70 ans sur la plus médiocre. Si l'on raisonne sur l'accroissement moyen entre 100 et 200 ans, on voit que l'écart entre volume grume et volume total est plus faible. Mais, surtout, les différences entre classes de fertilité sont bien moindres : de 4,9 à $6,5 \mathrm{~m}^{3} \mathrm{ha}^{-1} \mathrm{an}^{-1}$. Durant cette période, les chênaies les plus fertiles produisent en moyenne $4,5 \mathrm{~m}^{3} \mathrm{ha}^{-1} \mathrm{an}^{-1}$ de houppier, contre $2 \mathrm{~m}^{3} \mathrm{ha}^{-1} \mathrm{an}^{-1}$ pour les plus pauvres. Les bonnes capacités de production, au sens de la station, sont utilisées préférentiellement pour produire du houppier.

\subsection{Scénarios sylvicoles et ventilation de la production par catégories de diamètre}

Un des objectifs poursuivis par les partisans de la futaie irrégulière [25] est de minimiser la production de petits bois, tout en augmentant celle des gros bois les plus rémunérateurs pour le forestier. Il nous a semblé utile de mesurer quelle était réellement la production de petits bois d'une révolution de futaie régulière, de voir si elle dépend du traitement sylvicole et plus généralement de ventiler la production totale par catégorie de diamètre. Nous avons donc défini la production utile comme la production du volume bois-fort tige effectivement récolté : cela élimine notamment la mortalité et la production de branches. Pour les catégories de diamètre, nous reprendrons les classes usuelles :

- «Gros bois» $(\mathrm{GB})$ : classes de diamètre $50 \mathrm{~cm}$ et plus,

- «Bois moyens» (BM) : classes 30 à $45 \mathrm{~cm}$,

_ «Petits bois » (PB) : classes 20 et $25 \mathrm{~cm}$,

_ «Très petits bois » (TPB) : classes 5 à $15 \mathrm{~cm}$.

À chacune de ces catégories de bois correspondent des types d'usages très précis, d'où des prix unitaires qui sont très variables, ce qui explique l'intérêt des forestiers : bois de feu ou abandon (TPB, PB), bois reconstitué, par exemple par déroulage-collage (technique du Lamibois, PB), merrain, sciage (BM, GB), tranchage (GB). Parce que la valeur du produit augmente très fortement avec le diamètre, il est fondamental de savoir non seulement quelle proportion de la production est fournie par chaque produit, sur toute une révolution, mais aussi pendant quel laps de temps (qui détermine les rentrées financières et donc l'équilibre d'une forêt, d'un massif ou plus généralement d'une ressource).

Pour cela, nous nous intéressons aux 4 scénarios sylvicoles pour une même fertilité moyenne. La simulation du scénario «Quasi-TSF » constitue une très forte extrapolation au-delà du domaine de calibration du modèle ; pourtant, les résultats apparaissent raisonnables si on les compare aux quelques données dont nous disposons pour des arbres de taillis-sous-futaie (diamètre, hauteur, base du houppier, accroissement radial moyen). Les résultats sont présentés dans le tableau VI.

L'effet de la sylviculture sur la production en volume est bien différencié selon la définition considérée. Le volume bois-fort total (y compris les branches) montre une perte de production de $7 \%$ seulement pour le scénario «Très dynamique», $25 \%$ pour le «Quasi-TSF»; ces pertes sont beaucoup plus importantes pour le volume bois-fort tige ou le volume-grume : respectivement $-23 \%$ et -52 ou $-56 \%$. Ceci repose sur une répartition du volume différente : l'investissement dans le houppier représente $35 \%$ du total en peuplement très serré, contre $62 \%$ pour des arbres analogues aux réserves de taillissous-futaie. Conformément à l'observation courante, le modèle prédit des morphologies très trapues en «QuasiTSF » (diamètre $108 \mathrm{~cm}$, hauteur $30 \mathrm{~m}$, base du houppier $10 \mathrm{~m}$ ), très élancées en futaie classique (diamètre $65 \mathrm{~cm}$, hauteur $37 \mathrm{~m}$, base du houppier $17 \mathrm{~m}$ ). Sur une révolution complète, le volume de la plus haute valeur commerciale est divisé par deux entre un régime de futaie classique $\left(950 \mathrm{~m}^{3} / \mathrm{ha}\right)$ et la futaie très claire $\left(480 \mathrm{~m}^{3} / \mathrm{ha}\right)$. Simultanément, la maturité commerciale est nettement plus précoce, le diamètre de $60 \mathrm{~cm}$ étant atteint respectivement à 200 et 120 ans. Pour comparer l'opportunité économique de ces scénarios, il importe de tenir compte de l'ensemble des facteurs modifiés par les régimes d'éclaircies fortes : très forte perte en volume commercial, exploitabilité plus ou moins précoce, prix moins élevés pour des arbres dont la largeur des cernes varie entre 2,2 et $2,8 \mathrm{~mm}$ (contre une moyenne de 1,4 à $1,6 \mathrm{~mm}$ pour les régimes classiques, $1,9 \mathrm{~mm}$ pour le régime très dynamique).

Concernant la répartition par classes de diamètre, les produits de dimension inférieure ou égale à $25 \mathrm{~cm}$ représentent toujours 15 à $25 \%$ de la production totale, ce qui reste faible ; toutefois, le volume correspondant est de $300 \mathrm{~m}^{3} /$ ha en régime «Classique», contre $150 \mathrm{~m}^{3} /$ ha en «Quasi-TSF». Dans le même temps, les récoltes de bois moyens chutent de $330 \mathrm{~m}^{3} /$ ha à $90 \mathrm{~m}^{3} /$ ha. Cela est lié au fait que, lorsque les arbres arrivent à ce stade, l'essentiel de la population finale est déjà sélectionné en régime intensif. Il est frappant de constater que la production 
Tableau VI. Production des chênaies en fonction du régime sylvicole subi (définitions dans le texte) (fertilité moyenne, hauteur $25 \mathrm{~m}$ à 100 ans).

\begin{tabular}{|c|c|c|c|c|c|}
\hline Variable & Unité & $\begin{array}{c}\text { Pas de sylviculture } \\
\qquad R d i=1\end{array}$ & $\begin{array}{c}\text { Sylviculture } \\
\text { Classique } \\
\text { Rdi }=0,8\end{array}$ & $\begin{array}{l}\text { Très dynamique } \\
R d i=0,6\end{array}$ & $\begin{array}{l}\text { Quasi-TSF } \\
R d i=0,4\end{array}$ \\
\hline \multicolumn{6}{|c|}{ Production utile en volume bois-fort tige } \\
\hline Très petits bois $(\varnothing 5-15$ cm) & $\mathrm{m}^{3} \mathrm{ha}^{-1}$ & $\begin{array}{c}0 \\
\text { soit } 0 \%\end{array}$ & $\begin{array}{c}114 \\
\text { soit } 8 \%\end{array}$ & $\begin{array}{c}96 \\
\text { soit } 8 \%\end{array}$ & $\begin{array}{c}64 \\
\text { soit } 9 \%\end{array}$ \\
\hline Petits bois $(\varnothing 20-25 \mathrm{~cm})$ & $\mathrm{m}^{3} \mathrm{ha}^{-1}$ & $\begin{array}{c}0 \\
\text { soit } 0 \%\end{array}$ & $\begin{array}{c}206 \\
\text { soit } 15 \%\end{array}$ & $\begin{array}{c}100 \\
\text { soit } 8 \%\end{array}$ & $\begin{array}{c}80 \\
\text { soit } 11 \%\end{array}$ \\
\hline Bois moyens $(\varnothing 30-45 \mathrm{~cm})$ & $\mathrm{m}^{3} \mathrm{ha}^{-1}$ & $\begin{array}{c}0 \\
\text { soit } 0 \%\end{array}$ & $\begin{array}{c}328 \\
\text { soit } 23 \%\end{array}$ & $\begin{array}{c}232 \\
\text { soit } 19 \%\end{array}$ & $\begin{array}{c}89 \\
\text { soit } 12 \%\end{array}$ \\
\hline Gros bois ( $\varnothing 50 \mathrm{~cm}$ et plus) & $\mathrm{m}^{3} \mathrm{ha}^{-1}$ & $\begin{array}{c}780 \\
\text { soit } 51 \%\end{array}$ & $\begin{array}{c}728 \\
\text { soit } 52 \%\end{array}$ & $\begin{array}{c}766 \\
\text { soit } 64 \%\end{array}$ & $\begin{array}{c}510 \\
\text { soit } 69 \%\end{array}$ \\
\hline Mortalité & $\mathrm{m}^{3} \mathrm{ha}^{-1}$ & $\begin{array}{c}764 \\
\text { soit } 49 \%\end{array}$ & $\begin{array}{c}35 \\
\text { soit } 2 \%\end{array}$ & $\begin{array}{c}0 \\
\text { soit } 0 \%\end{array}$ & $\begin{array}{c}0 \\
\text { soit } 0 \%\end{array}$ \\
\hline \multicolumn{6}{|c|}{ Production totale de 0 à 200 ans } \\
\hline Volume bois-fort total Vtot7 & $\mathrm{m}^{3} \mathrm{ha}^{-1}$ & 1678 & 1602 & 1562 & 1259 \\
\hline Volume bois-fort tige Vtige7 & $\mathrm{m}^{3} \mathrm{ha}^{-1}$ & 1544 & 1412 & 1194 & 743 \\
\hline Volume de grume & $\mathrm{m}^{3} \mathrm{ha}^{-1}$ & 1088 & 957 & 837 & 484 \\
\hline $\begin{array}{l}\text { Pourcentage du houppier } \\
\text { dans la production totale }\end{array}$ & & $35 \%$ & $40 \%$ & $46 \%$ & $62 \%$ \\
\hline \multicolumn{6}{|c|}{ Production en pourcentage du scénario «Pas de sylviculture» } \\
\hline Vtot7 & & $100 \%$ & $95 \%$ & $93 \%$ & $75 \%$ \\
\hline Vtige7 & & $100 \%$ & $91 \%$ & $77 \%$ & $48 \%$ \\
\hline Vgrume & & $100 \%$ & $88 \%$ & $77 \%$ & $44 \%$ \\
\hline \multicolumn{6}{|c|}{ Phases de récolte en éclaircie par catégorie de produits : } \\
\hline Très petits bois $(\varnothing 5-15 \mathrm{~cm})$ & ans & & 20 à 110 & 20 à 60 & 20 à 60 \\
\hline Petits bois $(\varnothing 20-25 \mathrm{~cm})$ & ans & & 60 à 150 & 50 à 90 & 50 à 70 \\
\hline Bois moyens $(\varnothing 30-45 \mathrm{~cm})$ & ans & & 90 à 190 & 80 à 140 & 70 à 110 \\
\hline Gros bois ( $\varnothing 50 \mathrm{~cm}$ et plus) & ans & 200 & 160 à 190 & 130 à 160 & 110 à 140 \\
\hline \multicolumn{6}{|c|}{ Restent sur pied à 200 ans } \\
\hline Vtot7 & $\mathrm{m}^{3} \mathrm{ha}^{-1}$ & 902 & 815 & 904 & 688 \\
\hline Vtige7 & $\mathrm{m}^{3} \mathrm{ha}^{-1}$ & 780 & 652 & 626 & 343 \\
\hline Vgrume & $\mathrm{m}^{3} \mathrm{ha}^{-1}$ & 642 & 523 & 482 & 246 \\
\hline Hauteur dominante & $\mathrm{m}$ & 37,6 & 37,5 & 35,8 & 29,5 \\
\hline Base du houppier ( = $1^{\mathrm{er}}$ défaut $)$ & $\mathrm{m}$ & 18,3 & 17,4 & 15,3 & 10,3 \\
\hline Nombre de tiges par ha & $\mathrm{ha}^{-1}$ & 178 & 115 & 80 & 36 \\
\hline Diamètre moyen $D_{\mathrm{g}}$ & $\mathrm{cm}$ & 56,7 & 65,3 & 80,9 & 108,4 \\
\hline Diamètre dominant ${ }^{\circ} D_{0}$ & $\mathrm{~cm}$ & 60,1 & 66,9 & 80,9 & 108,4 \\
\hline
\end{tabular}

utile de gros bois est inchangée (700 à $800 \mathrm{~m}^{3} / \mathrm{ha}$ ) pour les 3 scénarios de futaie vraie, seule l'option «Quasi$\mathrm{TSF} »$ conduisant à une perte substantielle $\left(500 \mathrm{~m}^{3} / \mathrm{ha}\right)$.

On peut donc dire que, en termes de valeur relative, les gros bois pèsent le plus lourd, avec 50 à $70 \%$ de la production totale, tandis que les bois moyens accusent la plus forte diminution avec une intensification de la sylviculture. En termes bruts, l'orientation actuelle des forestiers vers une culture plus dynamique du Chêne [14] est susceptible de maintenir à son niveau actuel l'offre de gros bois, tout en diminuant d'un tiers la production de petits et moyens bois. Une sylviculture à très faible matériel sur pied conduit bien, conformément aux attentes des tenants de la futaie irrégulière, à une très forte concentration de la production, en valeur relative, sur les gros bois $(70 \%)$.

Si l'on considère maintenant la période (en gamme d'âges) durant laquelle les catégories de diamètre sont 
commercialisées, on constate que la vente des produits à très faible valeur (jusqu'au diamètre $25 \mathrm{~cm}$ ) ne se termine qu'à 150 ans en futaie classique, contre 90 et 70 ans en régimes dynamique et "Quasi-TSF». Peut-être plus encore que les volumes, ces chiffres plaident pour une intensification de la sylviculture aux stades les plus précoces possible. Sauf si de nouveaux marchés venaient à se développer pour ces bois, c'est presque les $2 / 3$ de la révolution qui sont affectés à une sylviculture sans recettes importantes. Bien sûr, on doit tempérer ce constat en considérant que, dans une futaie régulière à l'équilibre, les recettes dégagées par la vente des gros bois couvrent les dépenses au stade amélioration. Mais chacun sait que l'équilibre des classes d'âge est souvent très théorique et ne peut vraiment être atteint que sur une surface très vaste : en forêt communale, il nous semble indispensable de viser une meilleure rétribution des efforts et investissements (surtout après une longue phase de conversion) en accélérant la sylviculture juvénile.

Pour terminer, commentons le cas de l'absence de sylviculture. On peut imaginer que ce scénario s'étende soit sous l'effet d'une pression sociale hostile à toute forme d'exploitation forestière, soit comme conséquence d'une disparition de marchés rentables pour les petits bois. La première question soulevée est celle du maintien du Chêne, qui est très douteux sous cette hypothèse : dès que le climat convient au Hêtre (Normandie, Allier), on sait que sa dynamique lui permet d'envahir la Chênaie et de s'imposer en un laps de temps très court (40 ans pour atteindre la moitié de la surface terrière, dans l'essai de Réno-Valdieu [18]). Si l'on imagine que seul le stock final soit récolté, c'est $50 \%$ de la production qui serait recyclé sous forme de mortalité. Ce chiffre simple est intéressant par rapport aux questions soulevées par l'accroissement de l'effet de serre et par une autre demande sociale, visant à stocker du carbone en forêt. On voit qu'une activité sylvicole, même minimale, permet de stocker (au moins de façon transitoire, dans les produits forestiers) deux fois plus de carbone qu'une sylviculture sans éclaircies. En résumé, les chênaies offrent un exemple très frappant de la réponse que peuvent apporter des forestiers à la complexité des attentes sociales par rapport à la forêt (diversité génétique, stockage de carbone et impact psycho-sociologique de la gestion forestière).

\subsection{Ventilation du volume final selon différents compartiments}

La sylviculture des grands Chênes, sous ses diverses facettes (futaie et taillis-sous-futaie), revêt une grande importance pour l'aspect et l'évolution des territoires et des écosystèmes. Toutefois, d'un point de vue strictement économique, la rentabilité de l'hectare de Chênaie mériterait d'être mieux connue. Nous nous proposons d'apporter ici des éléments dendrométriques sur cette question ; pour cela, nous allons décomposer le volume de la population finale entre ses composantes purement géométriques, dont l'utilité dans les procédés de transformation est très variable.

Ce calcul est rendu possible par le logiciel Fagacées : nous donnons un exemple de sorties graphiques fournies par ce programme à la figure 6. On remarque que l'empattement est plus fort pour l'arbre à croissance très rapide, aussi bien en diamètre qu'en étendue verticale. La décroissance métrique de sa tige est également plus forte, aussi bien dans sa partie nette de branches que dans le houppier (cela se voit difficilement sur la figure, les échelles étant différentes) : le défilement sous le houppier s'établit, à 200 ans, aux valeurs respectives de $0,734-0,786-0,870$ et $1,060 \mathrm{~cm} / \mathrm{m}$ dans l'ordre des intensités d'éclaircie croissantes. La trace laissée à l'intérieur de la grume par l'élagage naturel diffère selon la sylviculture : pour les scénarios de futaie plus ou moins intensifs, cette trace est régulière (comme le processus d'élagage lui-même) ; pour le scénario «Quasi-TSF», la trace est plus incurvée, en lien avec une remontée pratiquement nulle du houppier pendant tout le développement de l'arbre. Ces différentes informations sur la géométrie interne peuvent être utilisées pour calculer des compartiments du volume.

La gamme des sylvicultures simulées produit des arbres de morphologies très différentes, comme nous l'avons signalé (tableau VII). Toutefois, quelques caractères internes sont presque insensibles à la sylviculture. Ainsi le nombre de cernes d'aubier, uniformément voisin de 20 (c'est un aspect que nous avions relevé sur notre échantillon INRA-ONF) : logiquement, la largeur d'aubier est donc plus importante chez les arbres à croissance rapide.

La répartition du volume total de l'arbre présente un effet sylvicole très net, avec 32 à $64 \%$ du volume dans le houppier selon l'intensité de la sylviculture. La valeur de $19 \mathrm{~m}^{3}$ de volume bois-fort total pour le scénario «Quasi$\mathrm{TSF}$ » pourra sembler exagérée, mais elle est confirmée par nos mesures de quelques arbres de taillis-sous-futaie très robustes.

Par rapport au volume total de la tige, le fait de considérer les découpes bois-fort ou $20 \mathrm{~cm}$ n'enlève qu'une fraction minime du volume, chez ces arbres mûrs. L'écorce représente toujours $14 \%$ du volume (le facteur d'écorce est uniforme) et l'aubier environ $20 \%$. La proportion de grume nette (déduction faite de l'écorce et l'aubier vers l'extérieur, du noyau central non élagué 


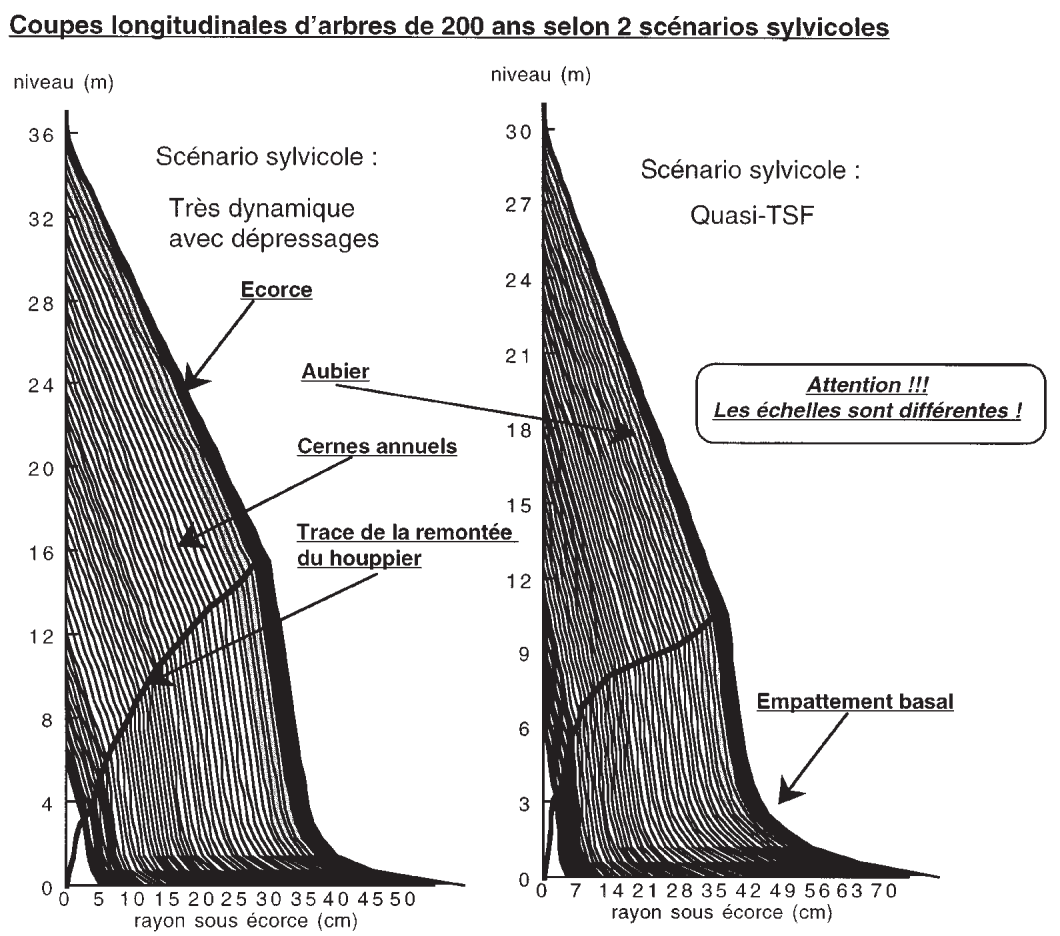

Figure 6. Exemples de coupes longitudinales d'arbres simulées par Fagacées. Les arbres correspondent à l'arbre moyen de la population des 70 plus gros par ha sur pied à 200 ans. Les échelles des diamètres et hauteurs sont adaptées à la morphologie de chaque arbre : elles sont donc différentes pour les 2 simulations. vers l'intérieur) varie peu entre traitements, de 41 à $44 \%$.

En conclusion, si l'on prend comme référence le volume de tige, le rendement théorique de nos Chênes lors de la transformation est peu sensible à la sylviculture et voisin de $40 \%$. Par rapport au volume bois-fort total, plus représentatif de la production biologique, l'effet sylvicole est très fort : dans l'ordre d'intensité croissante de la sylviculture, ces rendements s'établissent à 36, 34, 29 et $21 \%$.

Ces chiffres théoriques de rendement doivent être manipulés avec précautions. En effet, le rendement réel dépend d'autres facteurs que nous ne pouvons pas simuler pour l'instant : défauts liés à la ramification, gourmands, gélivure et autres défauts du matériau, fibre torse excessive. Quant au rendement en tranchage, il paraît très improbable que nous puissions le prédire de façon simple dans un avenir proche. Mais on peut sans doute considérer les résultats du tableau VII comme des indications maximales, purement géométriques, sur le volume de bois sain qu' on peut retirer d'une chênaie adulte.

Si l'on revient à des volumes par ha, la partie la plus hautement valorisée représente finalement, dans l'ordre d'intensité croissante des éclaircies, respectivement 157 , 199,235 et $141 \mathrm{~m}^{3} / \mathrm{ha}$, soit en pourcentage de la production totale $9,12,15$ et $11 \%$. Ce résumé très schématique néglige les récoltes intermédiaires : nous avons indiqué que 10 à $25 \%$ de la production totale, sous forme de bois moyens, peuvent être commercialisés à prix correct. Si l'on considère globalement les bois moyens et gros bois effectivement récoltés, on peut estimer leur équivalent en volume de bois de haute valeur (grume sous aubier et sans nœuds) à $300,390,380$ et $220 \mathrm{~m}^{3} / \mathrm{ha}$, soit en pourcentage de la production totale $18,24,24$ et $17 \%$.

En termes physiques, l'optimum se situe entre les scénarios «Classique» et «Très dynamique». Pour passer à un classement économique et financier, il faudrait tenir compte du diamètre (fortes différences de prix entre les diamètres 60 et $80 \mathrm{~cm}$ ), de l'accroissement radial (dépréciation éventuelle pour les bois ayant des cernes de 2,5 à $2,8 \mathrm{~mm}$ ) et de l'ensemble des coûts et recettes de la sylviculture (y compris le coût du temps). Ce travail d'optimisation reste à faire et pourrait se baser sur les indications physiques fournies ici.

La sylviculture du Chêne avec objectif tranchage est donc une activité assez optimiste, où l'on engage aujourd'hui des investissements dont le résultat est escompté dans 200 ans et où l'attention se polarise sur une petite fraction de la production biologique $(25 \%$ au maximum fourniront le bois d'œuvre sain, sans aubier et sans nœuds et quelques pourcents seulement le bois de tranchage). On comprend bien que l'état actuel du marché permet cette polarisation, par les prix très élevés accordés au merrain, au bois de tranchage et 
Tableau VII. Morphologie et répartition du volume, à 200 ans, dans l'arbre moyen de la population des 70 plus gros arbres par ha en fonction du régime sylvicole subi (définitions dans le texte) (fertilité moyenne, hauteur $25 \mathrm{~m}$ à 100 ans).

\begin{tabular}{|c|c|c|c|c|c|c|c|c|}
\hline & \multicolumn{2}{|c|}{ Pas de sylviculture } & \multicolumn{2}{|c|}{ Classique } & \multicolumn{2}{|c|}{ Très dynamique } & \multicolumn{2}{|c|}{ Quasi-TSF } \\
\hline diamètre $(\mathrm{cm})$ & \multicolumn{2}{|c|}{61,6} & \multicolumn{2}{|c|}{69,9} & \multicolumn{2}{|c|}{81,6} & \multicolumn{2}{|c|}{108,3} \\
\hline hauteur totale $(\mathrm{m})$ & \multicolumn{2}{|c|}{37,6} & \multicolumn{2}{|c|}{37,5} & \multicolumn{2}{|c|}{35,8} & \multicolumn{2}{|c|}{29,5} \\
\hline rayon du houppier (m) & \multicolumn{2}{|c|}{5,07} & \multicolumn{2}{|c|}{5,73} & \multicolumn{2}{|c|}{6,78} & \multicolumn{2}{|c|}{9,56} \\
\hline base du houppier (m) & \multicolumn{2}{|c|}{18,3} & \multicolumn{2}{|c|}{17,4} & \multicolumn{2}{|c|}{15,3} & \multicolumn{2}{|c|}{10,3} \\
\hline largeur d'aubier $(\mathrm{cm})$ & \multicolumn{2}{|c|}{2,81} & \multicolumn{2}{|c|}{3,25} & \multicolumn{2}{|c|}{3,75} & \multicolumn{2}{|c|}{5,44} \\
\hline nb de cernes d'aubier & \multicolumn{2}{|c|}{22} & \multicolumn{2}{|c|}{20} & \multicolumn{2}{|c|}{20} & \multicolumn{2}{|c|}{19} \\
\hline \multirow[t]{2}{*}{ largeur des cernes dans l'aubier (mm) } & & ,28 & & 62 & & 88 & & 86 \\
\hline & \multicolumn{8}{|c|}{ Répartition du volume bois-fort total $\left(\mathrm{m}^{3}\right.$ et $\left.\%\right)$} \\
\hline Bois-fort total & 6,18 & $100 \%$ & 8,29 & $100 \%$ & 11,53 & $100 \%$ & 19,10 & $100 \%$ \\
\hline Bois-fort tige & 5,15 & $83 \%$ & 6,44 & $78 \%$ & 7,95 & $69 \%$ & 9,52 & $50 \%$ \\
\hline Grume & 4,20 & $68 \%$ & 5,16 & $62 \%$ & 6,06 & $53 \%$ & 6,91 & $36 \%$ \\
\hline Bois-fort branches & 1,03 & $17 \%$ & 1,85 & $22 \%$ & 3,58 & $31 \%$ & 9,57 & $50 \%$ \\
\hline \multirow[t]{2}{*}{ Bois-fort houppier } & 1,98 & $32 \%$ & 3,13 & $38 \%$ & 5,48 & $47 \%$ & 12,19 & $64 \%$ \\
\hline & \multicolumn{8}{|c|}{ Répartition du volume dans la tige $\left(\mathrm{m}^{3}\right.$ et $\left.\%\right)$} \\
\hline Volume tige total & 5,16 & $100 \%$ & 6,44 & $100 \%$ & 7,95 & $100 \%$ & 9,52 & $100 \%$ \\
\hline Bois-fort tige (sur écorce) & 5,15 & $100 \%$ & 6,44 & $100 \%$ & 7,95 & $100 \%$ & 9,52 & $100 \%$ \\
\hline Tige découpe $20 \mathrm{~cm}$ (sur écorce) & 5,08 & $99 \%$ & 6,38 & $99 \%$ & 7,90 & $99 \%$ & 9,48 & $100 \%$ \\
\hline Grume (sur écorce) & 4,20 & $81 \%$ & 5,16 & $80 \%$ & 6,06 & $76 \%$ & 6,91 & $73 \%$ \\
\hline Volume sous écorce & 4,42 & $86 \%$ & 5,53 & $86 \%$ & 6,82 & $86 \%$ & 8,17 & $86 \%$ \\
\hline Ecorce & 0,74 & $14 \%$ & 0,92 & $14 \%$ & 1,13 & $14 \%$ & 1,36 & $14 \%$ \\
\hline Aubier & 1,06 & $21 \%$ & 1,30 & $20 \%$ & 1,59 & $20 \%$ & 2,02 & $21 \%$ \\
\hline Duramen & 3,36 & $65 \%$ & 4,23 & $66 \%$ & 5,22 & $66 \%$ & 6,15 & $65 \%$ \\
\hline Grume (sous aubier) & 2,87 & $56 \%$ & 3,53 & $55 \%$ & 4,18 & $53 \%$ & 4,70 & $49 \%$ \\
\hline Grume nette (- aubier - nœuds) & 2,24 & $44 \%$ & 2,84 & $44 \%$ & 3,36 & $42 \%$ & 3,92 & $41 \%$ \\
\hline
\end{tabular}

d'ébénisterie. Toutefois, on ne peut pas exclure qu'une évolution profonde des marchés et des exigences de la société vis-à-vis de sa forêt apporte un jour un surcroît de valeur (marchande, patrimoniale, stockage de carbone) aux 3/4 de la production qui sont aujourd'hui peu prisés, voire considérés comme un coût.

\section{DISCUSSION ET CONCLUSIONS}

Grâce à un important effort de recueil de données, auquel l'ONF a apporté un concours essentiel, nous avons pu mettre au point une méthode de prévision de la forme de la tige pour le Chêne sessile. Le modèle repose sur des principes simples : l'emboitement de deux troncs de cône, autour d'un point de jonction continu ou discontinu, qui correspond bien, en moyenne, à la base du houppier ; les 7 paramètres nécessaires pour caler cette fonction possèdent tous un sens forestier concret (hauteur totale, base du houppier, décroissance métrique, caractéristiques de l'empattement). La variabilité de ces paramètres synthétiques a pu être reliée simplement à quelques descripteurs de l'arbre : âge, diamètre et hauteur. Ceci permet d'estimer, à partir d'une information minimale sur l'arbre, comment son volume se répartit entre houppier et grume, tige et branches, tige à différentes découpes usuelles.

La méthode a été calée définitivement sur environ 750 Chênes adultes, provenant de placettes permanentes traitées en futaie. Une validation a été réalisée, en testant la qualité des estimations du volume bois-fort tige. Nous avons constaté que les biais et la variance des erreurs de prévision restaient très raisonnables, aussi bien pour des arbres jeunes (sauf si le diamètre est inférieur à $10 \mathrm{~cm}$ ) que pour des arbres très trapus de taillis-sous-futaie. Il n'y a pas de biais selon la provenance géographique ni selon la sylviculture. Notre méthode semble donc robuste et peut être appliquée avec confiance. Nous avons montré qu'elle a des performances équivalentes au tarif de cubage de [4], pour des arbres de futaie relativement élancés. Par contre, elle est plus générale et ne biaise pas le volume des sujets de taillis-sous-futaie.

La méthode a également permis de détecter un phénomène intéressant : à diamètre et hauteur fixés, la décroissance métrique de la tige nette de branches est beaucoup plus faible pour les arbres à houppier long. Or nos recherches récentes sur le phénomène de l'élagage naturel, menées en collaboration avec F. Colin (INRA, 
Croissance et Production, Champenoux), ont montré que la position de la première branche vivante est un caractère très variable à l'intérieur du peuplement. Cette forte variabilité est liée aux différences de port (fourches, grosses branches charpentières, branches fines à élagage rapide et continu), qu'on pourrait sans doute considérer comme aléatoires et traiter par des processus dynamiques stochastiques. Cela s'impose d'autant plus que les caractères liés à la ramification sont très importants en sylviculture (appréciation de la vigueur des arbres, choix des arbres d'avenir) et ont un effet important sur l'acquisition de la forme. Quoiqu'il en soit, nos méthodes de simulation actuelles ne permettent pas d'exploiter pleinement ce résultat, parce que la base du houppier est prédite comme un caractère à variation lente, aussi bien entre arbres qu'au cours du temps. Il y a là une piste pour des améliorations futures.

Au-delà de la forme de la tige, il est important de savoir prédire avec précision le volume de bois-fort total de l'arbre, branches comprises. Cette quantité donne accès à la production biologique primaire de l'écosystème, du moins pour sa partie aérienne ; elle permet aussi d'estimer la production de bois de houppier. Pour ce volume, nous avons constaté que le tarif de cubage de J. Bouchon peut être utilisé sans risques pour des arbres de futaie (pour lesquels il a été construit) mais sous-estime très nettement le volume des réserves de taillis-sousfutaie. Ce résultat, acquis sur quelques dizaines d'arbres, mériterait d'être plus largement validé, d'autant plus si l'on devait s'intéresser, dans les années à venir, à une meilleure mobilisation des bois en provenance de la forêt privée (voir le récent Rapport [2]). Dans l'immédiat, nous proposons une équation provisoire qui permet de rendre compte de l'énorme proportion de volume de houppier réalisée par les Chênes à croissance rapide.

L'ensemble de ces méthodes ont pu être intégrées dans un simulateur de la dynamique des chênaies et utilisées pour analyser la production en volume selon la fertilité de la station et selon 4 régimes d'éclaircie bien contrastés.

Des stations les plus médiocres aux plus riches (hauteurs dominantes à 100 ans variant de 17 à $32 \mathrm{~m}$ ), l'accroissement moyen du volume bois-fort total de 0 à 200 ans augmente ainsi de 5,2 à 10,3 $\mathrm{m}^{3} / \mathrm{ha} / \mathrm{an}$. Ces chiffres sont très élevés, si on les compare aux hypothèses retenues dans les aménagements. Mais nous avons montré qu'ils sont non-biaisés et que ce sont les hypothèses de gestion qui sous-estiment la possibilité en volume. C'est très clair sur un cas particulier : le Tarif Aménagement de Tronçais sous-estime le volume total, dans toutes les classes de diamètre, de $25 \%$.

Le pourcentage de la grume (volume géométrique de la tige jusqu'au premier défaut) dans la production totale s'établit à $55 \%$ pour un régime de futaie régulière classique, quelle que soit la station. Ce pourcentage varie par contre avec la sylviculture : avec un scénario de futaie très claire, assez proche du taillis-sous-futaie par ses résultats, il descend à $40 \%$. Ces chiffres expliquent pourquoi des futaies adultes assez denses n'offrent que $500 \mathrm{~m}^{3} /$ ha de volume commercialement utile, malgré un volume total élevé (800 à $900 \mathrm{~m}^{3} / \mathrm{ha}$ ).

Nous pouvons également chiffrer assez précisément les différents effets sur la production des scénarios à croissance rapide : par rapport à la sylviculture prudente pratiquée actuellement en futaie régulière, un traitement en futaie très claire (surface terrière 10 à $20 \mathrm{~m}^{2} / \mathrm{ha}$ ) permet une croissance en diamètre plus rapide de $50 \%$ (diamètre de $108 \mathrm{~cm}$ contre $70 \mathrm{~cm}$ à $200 \mathrm{ans}$ ); en revanche, il entraine une croissance en hauteur plus faible (déficit estimé ici à $8 \mathrm{~m}$ à 200 ans), une grume limitée à $10 \mathrm{~m}$ (contre 17) ; la perte en volume total n'est que de $20 \%$, grâce à la branchaison exubérante, mais le volume commercial chute de moitié $\left(250 \mathrm{~m}^{3} /\right.$ ha contre 500$)$.

Nous avons cherché à utiliser plus finement le simulateur, en décomposant la récolte finale entre divers compartiments : houppier, écorce, aubier, noyau central de la tige contenant des nœuds. La soustraction progressive de ces différents compartiments reproduit en fait un processus de transformation industrielle. Au stade de l'exploitation, l'acheteur doit se débarasser des houppiers (30 à $60 \%$ du volume, selon la morphologie des arbres). Puis la grume restante est triée en bille et surbilles : les industries vont alors transformer un volume géométrique correspondant au duramen de la grume (écorce et aubier sont purgés, soit encore $30 \%$ du volume de la grume sur écorce). Enfin le marché du bois va accorder une très nette surprime à la bille de pied, où les défauts d'élagage sont minimes : nous estimons cette surprime en considérant le volume de la grume nette de nœuds, ce qui revient à enlever $20 \%$ du volume-grume sous aubier. Le rendement industriel final d'un $\mathrm{m}^{3}$ de Chêne est donc forcément faible : $34 \%$ du volume pour un arbre de futaie classique, $21 \%$ pour une réserve de taillis-sous-futaie.

L'ensemble de ces résultats permet de revenir au problème initial, à savoir la disparité entre les volumes «recherche» et «gestion». Le rendement lors des première et seconde transformation du Chêne - et plus généralement des feuillus par rapport aux résineux - est faible et les déchets (houppier et pertes de matière) ont une très faible valeur marchande. Quel que soit leur volume (il peut être énorme), ces déchets pèsent très peu dans les transactions et dans la formation du prix d'une coupe contenant sciage, merrain, tranchage. Sous la pression des acheteurs, qui est la plus forte chez ceux qui recherchent les gros bois, les forestiers sont sans doute amenés à cuber les coupes jusqu'au premier défaut. Ils sont donc 
accoutumés à considérer comme la production d'une parcelle celle qui se vend le mieux, c'est-à-dire le volume-grume, et finissent logiquement par trouver démesurés des chiffres de $800 \mathrm{~m}^{3} /$ ha annoncés par les chercheurs. Le désaccord sur les volumes est donc le plus élémentaire qui soit, à savoir une différence sur la définition de l'objet cubé. La vente de produits façonnés bord de route fait disparaître ces ambiguïtés. Dans le cas des ventes sur pied, une meilleure séparation, assise sur des barêmes plus précis, entre le volume-grume et le volume de houppier assurerait plus de transparence aux transactions. Il nous semble aussi souhaitable de ne pas sous-estimer le volume de houppier et, pour cela, de ne pas l'estimer comme un pourcentage du volume-grume. Enfin, lorsque la discussion se porte sur la productivité des forêts, en particulier sur son augmentation à long terme, les tarifs de cubage «recherche» devraient s'imposer exclusivement, dès lors que leur robustesse a été établie pour la ressource en question (futaies, taillissous-futaie).

Remerciements : Cette recherche a été financée par le contrat INRA-ONF «Sylviculture et qualité du bois de Chêne sessile», 1992-96 (ccordinateur G. Nepveu) ainsi que par l'Union Européenne, Contrat OAKKEY No. CT 95-0823, 1996-2000 (coordinateur F. Colin). La description des stations a été effectuée par nos collègues J.M. Gilbert (CEMAGREF Nogent/Vernisson) et Y. Lefèvre (INRA Nancy), que nous remercions. Les mesures, saisies et premières analyses ont été réalisées avec la participation d'André Perrin, Yannick Le Doré, Claire Alexandre, Sonia Saïd et Paloma Lopez-Izquierdo Botin. Nous remercions enfin les deux relecteurs anonymes de la revue pour leurs critiques et suggestions.

\section{RÉFÉRENCES}

[1] Arnal M., Drouineau S., Évaluation de la productivité et réalisation de normes de sylviculture Chêne et Hêtre en Normandie, Mémoire stage de fin d'études FIF, ENGREFNancy 1997, p. 80.

[2] Bianco J.L., La forêt : une chance pour la France, Rapport au Premier Ministre, Paris 1998, p. 120.

[3] Bouchon J., Norme provisoire pour le Chêne de qualité du secteur ligérien, Doc. int. Station de Sylviculture et Production, INRA, Champenoux 1970, p. 13.

[4] Bouchon J., Les tarifs de cubage, INRA et ENGREF, Nancy (France) 1974, p. $57+$ annexes.

[5] Bulletin Technique de 1'ONF, $N^{\circ}$ spécial Chêne, 33, 1997, p. 107.

[6] Colin F. et al., Rapport Annuel d'avancement du Contrat Européen OAKKEY CT95-0823, INRA, Nancy-Champenoux, France 1998, p. 183.

[7] Dhôte J.F., de Hercé E., Un modèle hyperbolique pour l'ajustement de faisceaux de courbes hauteur-diamètre, Can. J. For. Res. 24, 9 (1994), 1782-1790.
[8] Dhôte J.F., E. Hatsch, D. Rittié, Profil de la tige et géométrie de l'aubier chez le Chêne sessile (Quercus petraea Liebl.), Bull. Techn. ONF 33 (1997), 59-82.

[9] Dreyfus Ph., Bonnet F.R., Capsis : logiciel de simulation de conduites sylvicoles, Rev. For. Française, XLVII, $n^{\circ} \mathrm{sp}$. 1995, p. 111-115.

[10] Duplat P., Tran-Ha M., Modélisation de la croissance en hauteur dominante du Chêne sessile (Quercus petraea Liebl.) en France. Variabilité inter-régionale et effet de la période récente (1959-1993), Ann. Sc. For. 54 (1997), 611-634.

[11] Hatsch E., Géométrie de l'aubier et des accroissements ligneux dans les tiges de jeunes Chênes sessiles (Quercus petraea (Matt.) Liebl.), DEA de Biologie Forestière. ENGREF Nancy, sept. 1993, p. $25+$ annexes.

[12] Hatsch E., Répartition de l'aubier et acquisition de la forme de la tige chez le Chêne sessile ( $Q$. petraea (Matt) Liebl.). Thèse de doctorat, ENGREF 1997, p. 170 + annexes.

[13] Houllier F., Leban J.M., Colin F., Linking growth modelling to timber quality assessment for Norway spruce, For. Ecol. Man. 74 (1995), 91-102.

[14] Jarret P., Sylviculture du Chêne sessile, Bull. Techn. ONF 31 (1996), 21-28.

[15] Nepveu G. et al., Rapport annuel de la Convention INRA-ONF «Sylviculture et qualité du bois de Chêne», INRA, Champenoux-Nancy (France) 1996, p. 220.

[16] Nepveu G., Guilley E., Étude et modélisation de la qualité du bois de chêne sessile (Quercus petraea Liebl) selon la vitesse de croissance, Bull. Techn. ONF 33 (1997), 83-91.

[17] Nepveu G., Dhôte J.F., Sylviculture et Qualité du bois de Chêne sessile, Rapport Final du Contrat INRA-ONF 199296. INRA, Champenoux (France) 1998, p. 68.

[18] Ningre F., Comparaison de différentes modalités d'éclaircie du Chêne sessile. Premiers résultats d'un dispositif expérimental situé en forêt domaniale de Réno-Valdieu (Orne), Rev. For. Fr. XLII 2 (1990), 254-264.

[19] O.N.F., Aménagement de la Forêt de Tronçais. Plan de Gestion, ONF, Moulins (Allier), France 1974, p. 65

[20] Pardé J., Table de production pour les forêts de chêne rouvre de qualité tranchage du secteur ligérien, Notes Techniques Forestières, 11, ENGREF-INRA, Nancy (France) 1962, p. 6.

[21] Planchais I., Modélisation de la croissance et de l'architecture du jeune Hêtre (Fagus silvatica L.) : effet de l'éclairement, Thèse de Doctorat, Université de Paris-SudOrsay, France 1998, p. 199 + annexes.

[22] Polge H., Keller R., Qualité du bois et largeur d'accroissements en Forêt de Tronçais, Ann. Sc. Forest. 30, 2 (1973), 91-125.

[23] Pressler R., Das Gesetz der Stammbildung, Arnoldische Buchhandlung (Leipzig) 1865, p. 153.

[24] Reineke, L.H., Perfecting a stand-density index for even-aged forests, J. Agric. Res. 46, 7 (1933), 627-638.

[25] Turkheim B. de, La sylviculture préconisée par Pro Silva : théorie et pratique, Forêt-entreprise 113 (1997), 14-15. 